\title{
SPACECRAFT CHARGING MODELING - NASCAP-2K 2014 Annual Report
}

V. A. Davis and M. J. Mandell

Leidos

10260 Campus Point Drive, Mailstop C4

San Diego, CA 92121

19 September 2014

Technical Report

APPROVED FOR PUBLIC RELEASE; DISTRIBUTION IS UNLIMITED.

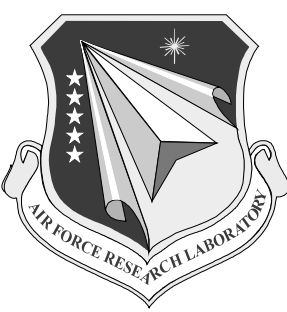

AIR FORCE RESEARCH LABORATORY Space Vehicles Directorate 3550 Aberdeen Ave SE AIR FORCE MATERIEL COMMAND KIRTLAND AIR FORCE BASE, NM 87117-5776 


\section{DTIC COPY}

\section{NOTICE AND SIGNATURE PAGE}

Using Government drawings, specifications, or other data included in this document for any purpose other than Government procurement does not in any way obligate the U.S. Government. The fact that the Government formulated or supplied the drawings, specifications, or other data does not license the holder or any other person or corporation; or convey any rights or permission to manufacture, use, or sell any patented invention that may relate to them.

This report was cleared for public release by the 377 ABW Public Affairs Office and is available to the general public, including foreign nationals. Copies may be obtained from the Defense Technical Information Center (DTIC) (http://www.dtic.mil).

AFRL-RV-PS-TR-2014-0188 HAS BEEN REVIEWED AND IS APPROVED FOR

PUBLICATION IN ACCORDANCE WITH ASSIGNED DISTRIBUTION STATEMENT.

//SIGNED//

Adrian Wheelock

Project Manager, AFRL/RVBXR
/ /SIGNED//

Glenn M. Vaughan, Colonel, USAF

Chief, Battlespace Environment Division

This report is published in the interest of scientific and technical information exchange, and its publication does not constitute the Government's approval or disapproval of its ideas or findings. 


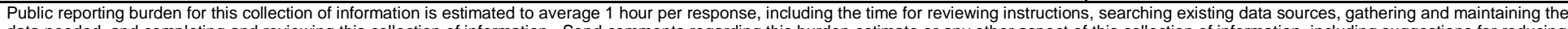

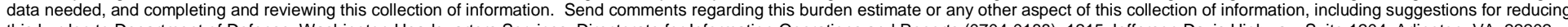

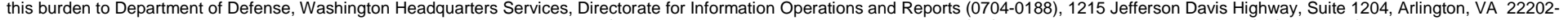

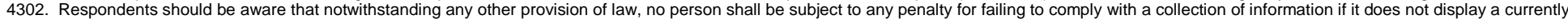
valid OMB control number. PLEASE DO NOT RETURN YOUR FORM TO THE ABOVE ADDRESS.

\begin{tabular}{|l|l|l}
\hline 1. REPORT DATE (DD-MM-YYYY) & 2. REPORT TYPE & 3. DATES COVERED (FrOm - To)
\end{tabular}

19-09-2014 $\quad$ Technical Report

\section{TITLE AND SUBTITLE}

Spacecraft Charging Modeling-Nascap- $2 k$

2014 Annual Report

\section{Sep 2013 to 19 Sep 2014}

5a. CONTRACT NUMBER

FA9453-11-C-0262

5b. GRANT NUMBER

5c. PROGRAM ELEMENT NUMBER

$62601 \mathrm{~F}$

5d. PROJECT NUMBER

1010

V. A. Davis and M. J. Mandell

5e. TASK NUMBER

PPM00012836

5f. WORK UNIT NUMBER

EF003277

8. PERFORMING ORGANIZATION REPORT NUMBER

Leidos

10260 Campus Point Drive, Mailstop C4

San Diego, CA 92121

9. SPONSORING I MONITORING AGENCY NAME(S) AND ADDRESS(ES)

Air Force Research Laboratory

10. SPONSOR/MONITOR'S ACRONYM(S)

AFRL/RVBXR

Space Vehicles Directorate

3550 Aberdeen Avenue SE

Kirtland AFB, NM 87117-5776

\section{SPONSOR/MONITOR'S REPORT NUMBER(S)}

AFRL-RV-PS-TR-2014-0188

\section{DISTRIBUTION I AVAILABILITY STATEMENT}

Approved for public release; distribution is unlimited. (377ABW-2014-1024 dtd 02 Jan 2015)

\section{SUPPLEMENTARY NOTES}

\section{ABSTRACT}

In support of the larger goal to provide a plasma engineering capability to the spacecraft community, the objective of the Spacecraft Charging Modeling - Nascap-2k contract is to develop, incorporate, test, and validate new algorithms for the three-dimensional plasmaenvironment spacecraft interactions computational tool, Nascap-2k. Nascap-2k is being modified to extend the range of plasma physics phenomena that the code can simulate, make the advanced code capabilities more accessible to users, and improve and maintain both the graphical and non-graphical interfaces to the code. The upgraded code is being used to simulate problems of interest to AFRL.

During the third year, Nascap-2k 4.1 was modified to be more user-friendly and address a wider array of charging problems.

\section{SUBJECT TERMS}

Nascap-2k, Potentials, Space Environment, Spacecraft, Spacecraft Charging

\begin{tabular}{|c|c|c|c|c|c|}
\hline \multicolumn{3}{|c|}{ 16. SECURITY CLASSIFICATION OF: } & \multirow{2}{*}{$\begin{array}{r}\text { 17. LIMITATION } \\
\text { OF ABSTRACT } \\
\text { Unlimited }\end{array}$} & \multirow{2}{*}{$\begin{array}{l}\text { 18. NUMBER } \\
\text { OF PAGES } \\
\qquad 52\end{array}$} & \multirow{2}{*}{$\begin{array}{l}\text { 19a. NAME OF RESPONSIBLE PERSON } \\
\text { Adrian Wheelock } \\
\text { 19b. TELEPHONE NUMBER (include area } \\
\text { code) }\end{array}$} \\
\hline $\begin{array}{l}\text { a. REPORT } \\
\text { Unclassified }\end{array}$ & $\begin{array}{l}\text { b. ABSTRACT } \\
\text { Unclassified }\end{array}$ & $\begin{array}{l}\text { c. THIS PAGE } \\
\text { Unclassified }\end{array}$ & & & \\
\hline
\end{tabular}


This page is intentionally left blank.

Approved for public release; distribution is unlimited. 


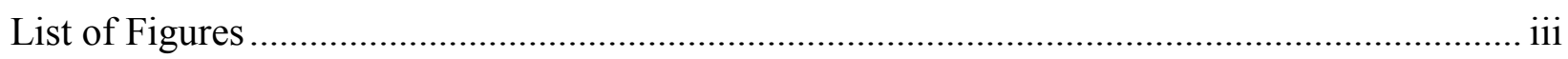

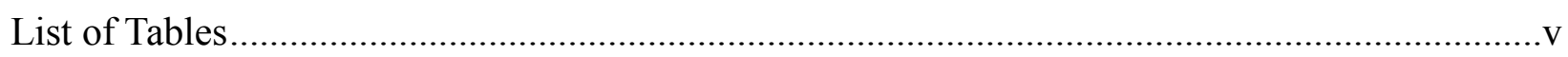

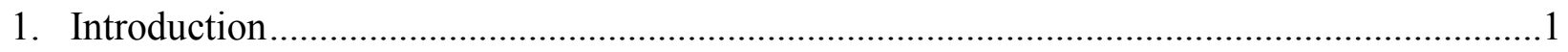

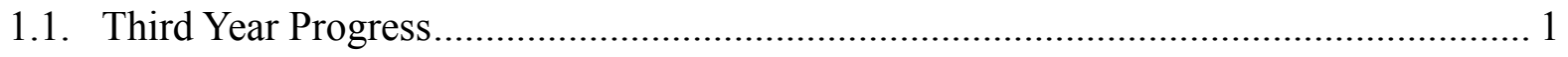

1.1.1. Maintenance and Support .......................................................................... 1

1.1.2. Charging Index.......................................................................................... 4

1.1.2.1. SEE Spacecraft Charging Handbook ..................................................... 4

1.1.2.2. Charging in a LEO Plasma ............................................................. 5

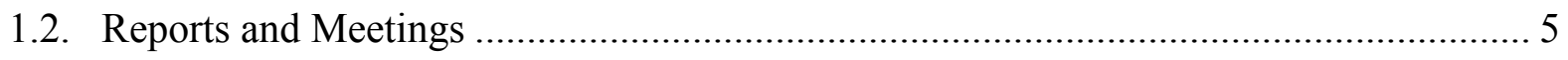

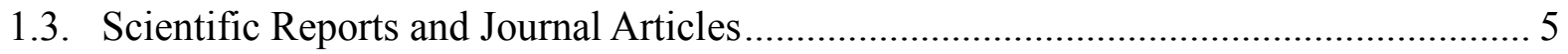

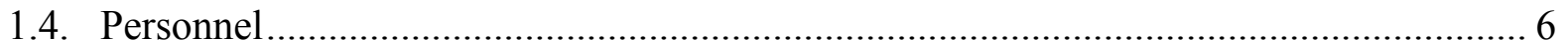

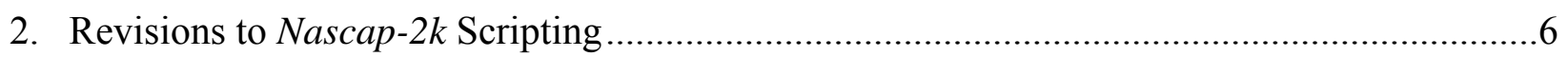

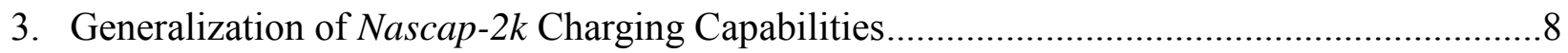

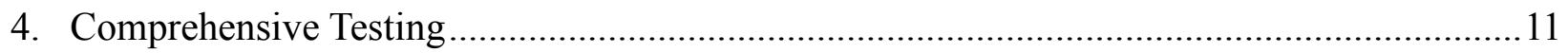

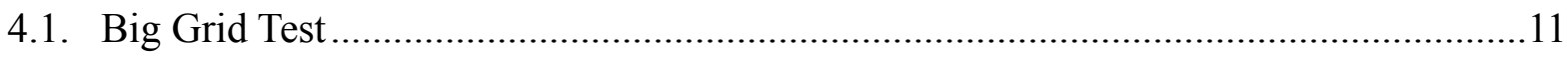

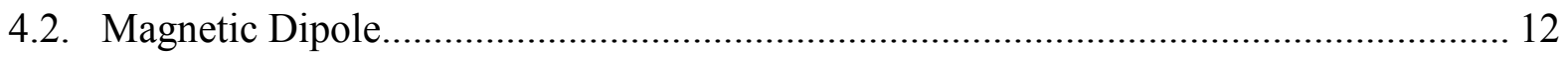

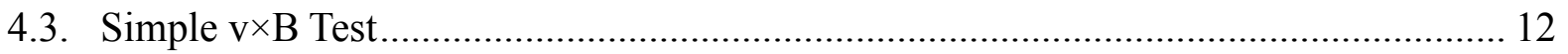

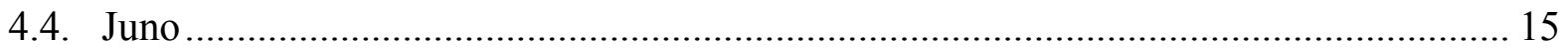

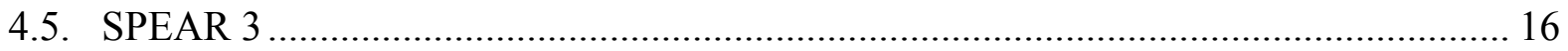

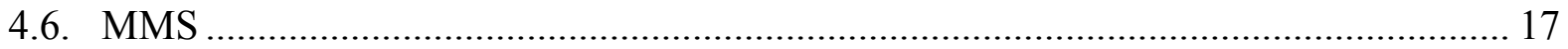

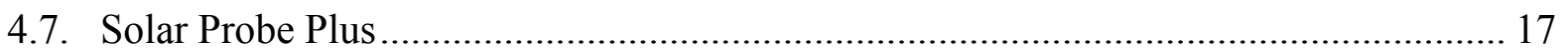

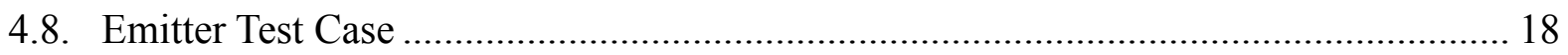

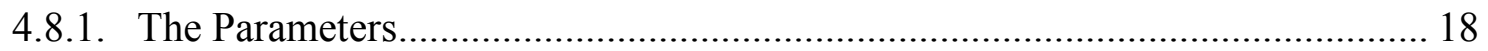

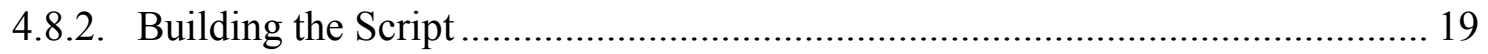

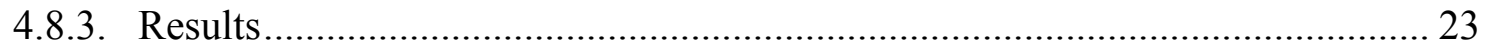

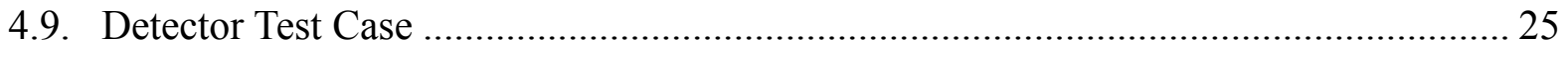

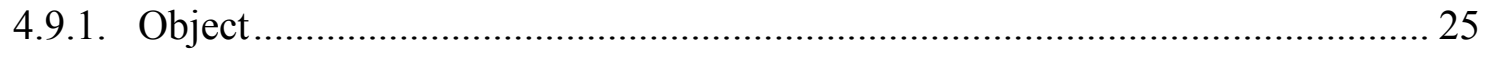

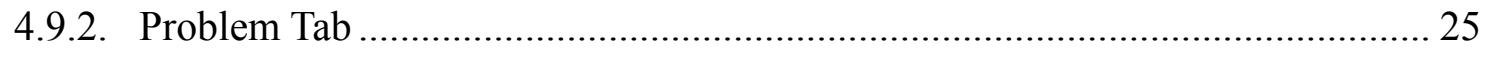

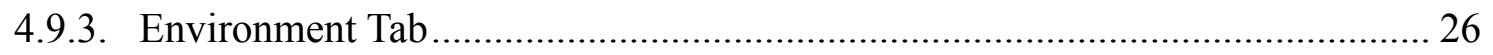

4.9.4. Applied Potentials Tab ............................................................................ 26

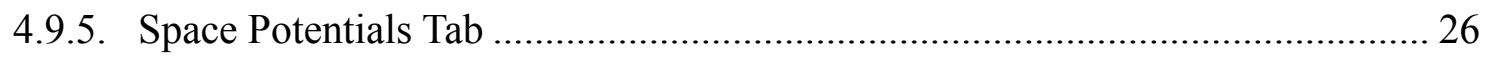




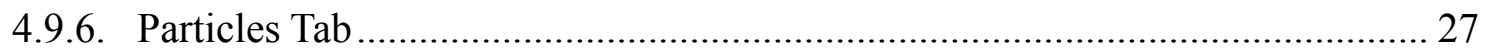

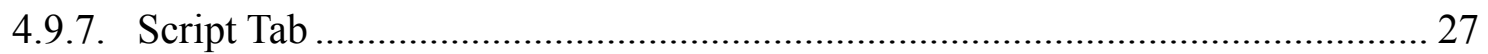

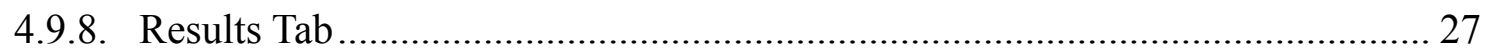

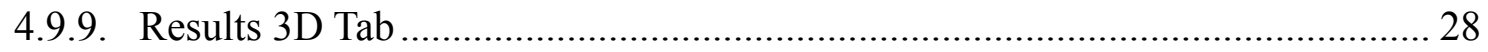

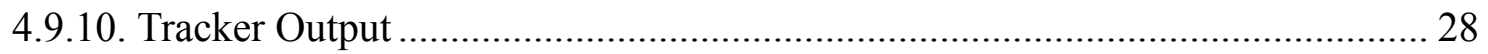

5. Memo Summarizing Resources for Internal Charging Computations...................................30

6. Memo Summarizing Resources for Auroral Charging Computations ...................................33

7. Semi-analytic and PIC (Particle-in-cell) Methods for Quantifying Charging in Dense, Cold Plasma... 


\section{List of Figures}

Page

Figure 1. Adding the FILE protocol to the "Exception Site List." .5

Figure 2. Comparison of Scripts Used to Compute Charging in a Geosynchronous Substorm using the Old and New Schemes

Figure 3. Comparison of Scripts Used to Compute Potentials in Space Given Specified

Surface Potentials Using the Old and New Schemes

Figure 4. Comparison of Scripts Used to Specify the DSX Charging Problem Using the

Old and New Schemes 8

Figure 5. Geometry and Materials for First Test Case for Charging in LEO with Analytic Currents from a Convected Maxwellian Distribution.............................................................. 10

Figure 6. Potentials Resulting from Charging Calculation using Geometry of Figure 5..............11

Figure 7. Potentials about -10 V Parallelepiped in a $4 \mathrm{~cm}$ Debye Length Plasma ......................12

Figure 8. Electron Trajectories toward a $+10 \mathrm{~V}$ Sphere with a Magnetic Dipole at Its Center (Potentials in a Plane through the Center of the Sphere Are Also Shown) ......................12

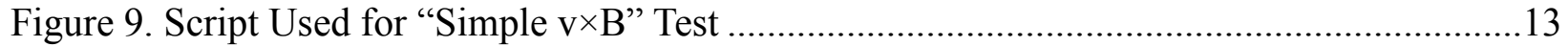

Figure 10. Object Toolkit Object Used in Verification Calculation ........................................... 14

Figure 11. Final (Steady State) Surface Potentials for the Verification Calculation.....................14

Figure 12. Absolute and Differential Potential as a Function of Time (and Density) at Selected Insulating Surface Elements (Two Surfaces from Each Insulating Collar are Shown)

Figure 13. Charging Current to Insulating Elements at Each End of the Cylinder as a Function of Time (and Density)

Figure 14. Surface Potentials on Juno for the Bottom (Conductive) Side (Left) and the Top (Insulating Solar Cell) Side (Right).

Figure 15. Potentials about the SPEAR III Model, with Parameters as Given in Text. 17

Figure 16. Aluminum Cylinder Showing Emitter Surface Element 18

Figure 17. Environment Parameters 19

Figure 18. Emitter Parameters .19

iii

Approved for public release; distribution is unlimited. 
Figure 19. Particles Tab Specification for Emitter Electrons......................................................20

Figure 20. Editing of Script to Simplify Electron Emitter Files ...................................................21

Figure 21. Particles Tab Specification for Sheath Electrons .......................................................22

Figure 22. Script with Duplicated Create Particles Commands ………….....................................22

Figure 23. Editing of a Pair of Create Particles Commands to Create both Emitter and

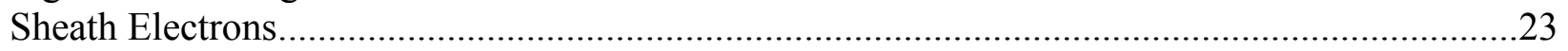

Figure 24. Edit of Tracker Input file. Note That Both Species (Sheath Electrons and

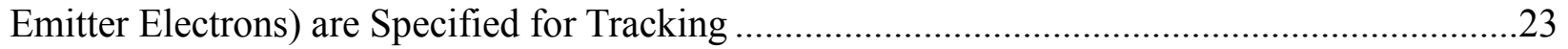

Figure 25. Potential versus Time for the Emitter Problem .........................................................24

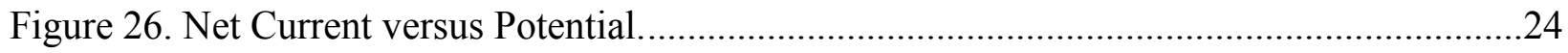

Figure 27. Charging Dynamics with the Stabilizing Current Derivatives Set to Zero ...................25

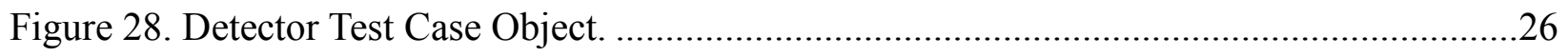

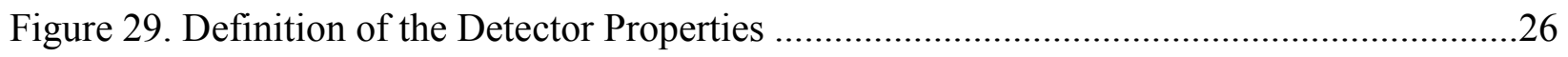

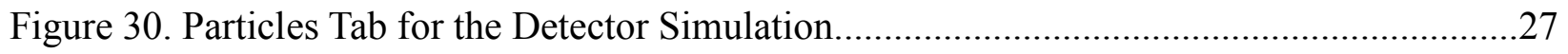

Figure 31. Script for the Detector Problem (Default Script) ………...........................................2

Figure 32. Results Tab Following Execution of the Detector Problem .......................................28

Figure 33. Results 3D Tab, Showing Properties of Detector Surface Element, and Showing Bowing Out of Potentials through the Collimator............................................................22

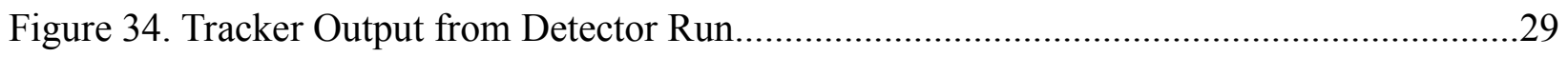




\section{List of Tables}

Page

Table 1. Charging Calculations Allowed by Default ...............................................................

Table 2. Calculated Currents to the SPEAR III Model for Parameters Given in Text and for Three Magnetic Field Configurations .........................................................................17 
This page is intentionally left blank. 


\section{Introduction}

In support of the larger goal to provide a plasma engineering capability to the spacecraft community, the objective of the Spacecraft Charging Modeling-Nascap-2k contract is to develop, incorporate, test, and validate new algorithms for the three-dimensional plasmaenvironment spacecraft interactions computational tool, Nascap- $2 k$. Nascap- $2 k$ is being modified to extend the range of plasma physics phenomena that the code can simulate, make the advanced code capabilities more accessible to users, and improve and maintain both the graphical and nongraphical interfaces to the code. The upgraded code is being used to simulate problems of interest to AFRL, including support of the DSX (Demonstration and Science eXperiments) spacecraft and program.

\subsection{Third Year Progress}

Details on progress during the period from September 21, 2013 through September 19, 2014 are given in the following sections. Key accomplishments included in Version 4.2 are the following:

- Nascap-2k has been made significantly more user friendly. The scripting was revised to make the commands more intuitive. The interface was modified to be more automated and intuitive.

- The Nascap-2k charging capability was generalized to address a wider array of charging problems, including charging in dense plasma.

- Nascap-2k 4.2 was prepared for release, including updating the documentation.

\subsubsection{Maintenance and Support}

The Nascap-2k scripting for Version 4.2 was revised to make the functionality of the commands more intuitive:

- A new primary script command Read Object was added to replace the "ReadObject" subcommand of the Charge Surfaces script command. It also initializes surface potentials.

- The subcommands of the Charge Surfaces command that were used to perform initialization and database access operations were eliminated. These functions are now performed automatically as needed.

- The subcommands used to set calculational parameters were combined into a single "Set Parameters" subcommand.

- Old scripts still run correctly.

- Additional discussion of these revisions appears in Section 2.

The Nascap-2k charging capability for Version 4.2 was generalized:

- The graphical user interface and the default scripts were revised to allow for a wider variety of charging problems to be easily specified. The underlying coding was streamlined at the same time. 
- The ability to compute and use secondary electron currents in all charging calculations with tracked currents was added. The yield is computed using analytic currents with the correct ion masses.

- The portions of the code that compute the current to a surface and its derivative with respect to potential were revised to be more transparent and easier to maintain. Comments were added, the code reorganized, and duplicate coding removed.

- Additional discussion of the generalization of the Nascap-2k charging capability appears in Section 3.

The Version 4.2 interface was modified to make the code easier to use.

- The ability to display the differential potential on the Results and Results 3D tabs was added.

- Earlier a temporary button had been added to the Nascap-2k Edit Materials dialog box that allowed the user to reset the material properties to their default values. The label on this button was changed to "Reset to Default Properties" and its functionality was expanded to also immediately redisplay the dialog box with the revised values.

- The lists of quantities available on the pull down menus on the Results and Results 3D tabs were made dynamic. The items in the pull down menus are now only those quantities available in the database for the presently displayed time step.

- The names of the more obscure spatial quantities available for plotting were revisited and the names of several quantities on the pull down menu on the Results 3D tab were revised.

- Object Toolkit, GridTool, and the Nascap-2k graphical user interface were modified to be more tightly coupled.

o GridTool and Object Toolkit now automatically load the object when launched from the Nascap- $2 k$ graphical user interface.

o Nascap-2k now automatically loads the revised object when returning from Object Toolkit.

o When called from Nascap-2k, GridTool now creates the grid file with the right name in the right place by default.

- When an object is read into Nascap-2k, all of the pull down lists of materials and conductors are immediately updated.

- The number of iterations and the number of timesteps are now the same variable.

- The underlying code and the user interface were modified so that the units in which charge density is displayed on the Nascap-2k Results 3D tab is now number per cubic meter.

- The Nascap-2k user interface was modified so that potentials specified on the Applied Potentials tab propagate to the first instance in the script and the reverse.

- The ability to display the time history of current components was added to Results tab coding.

Additional improvements to the default environments, the algorithms, and the output files made during this period include the following: 
- A new predefined geosynchronous environment based on an environment measured by SCATHA was added.

- The predefined Freja auroral environments were modified to exclude the unphysical low energy power law electrons.

- In Object Toolkit, nodes of the same component that are within a specified distance of each other are automatically combined. This distance was changed from a fixed value of one millimeter $(0.001 \mathrm{~m})$ to a user specifiable parameter.

- The maximum length of a database name was determined to be 70 characters.

- The charging code was modified so that charging calculations will execute without an environment and even without an object.

- The script interpretation code was modified so that multiple "TimeParams" folders in a "DoTimeSteps" subcommand can be used to specify a sequence of steps with different timestep length parameters to be used in a single charging calculation.

- The values printed in Tracker text output files in reverse trajectory calculations were modified so that they have physical meaning. Macroparticles carry current without the Maxwellian exponential factor. The values printed as the current to object, lost current, and collected current now include an exponential factor appropriate to the final velocity of the macroparticle. This means that the "lost current" is the current actually collected by the object and the other values are related to the current.

- The particle specification code was modified so that the emitter current is assigned to the surface element which is emitting the current.

- The particle specification code was modified to allow for injected current from the computational grid boundary in particle-in-cell (PIC) calculations when the electric field is outward.

- The original implementation of the zero total current algorithm is only effective if all the currents are analytic. The implementation was revised so that the algorithm can be used in charging calculations with tracked currents.

Nascap-2k 4.2 is being prepared for release:

- The new Nascap-2k functionality was fully tested. A number of small issues were identified and the code was modified as appropriate. A discussion of this testing is included in Section 4. Example problems using the new emitter and detector capabilities are included in Sections 4.8 and 4.9 below.

- The Nascap-2k documentation, including the examples in Nascap-2k User's Manual and the on-line documentation, was updated. It is now undergoing final revision.

- The JavaChart source code (used to create and display the graph on the Results tab) was added to the Nascap-2k user interface Eclipse project and archived in the Source Safe database. This eliminates the extra javachart.jar file included in the distribution and will insure that this code will remain compatible with future revisions of Java.

- The Nascap-2k 4.2 Beta code was sent to AFRL and three additional beta testers: Dr. Michelle Donegan of Applied Physics Laboratory Johns Hopkins University, Wousik Kim of the Jet Propulsion Laboratory, and Nikki Noushkam of Orbital Sciences Corporation.

Development of Nascap-2k 4.3 has begun with the following two activities: 
- Under separate funding, the potential solution module was parallelized. As part of that effort, the code was modified to dramatically reduce reading and writing to the database. This change speeds up the code from negligible to $50 \%$ faster depending on the number of special elements, the number of grids, and the complexity of the grid structure.

- We moved the Nascap-2 $k$ source code to the latest development environment (Microsoft Visual Studio 2013) and compilers (Microsoft Visual C++ 2013 and Intel Visual Fortran 14). We successfully ran the standard test cases. With the new compilers, the code runs about $10 \%$ faster.

\subsubsection{Charging Index}

Resources regarding internal charging environments and internal charging computation were identified and summarized. This memo is included as Section 5 below.

Resources regarding auroral charging environments and auroral charging computation were identified and summarized. This memo is included as Section 6 below.

We began an investigation of DMSP charging events. We reviewed the literature for interesting charging events. We requested and received environment and operations data for two events. We examined the time history for these events.

\subsubsection{SEE Spacecraft Charging Handbook}

In order to support its use in the AFRL Charging Index study, we modified the NASA code, SEE Spacecraft Charging Handbook, so the code does not fail on browser version verification. Layout issues on the 3-D charging pages remain. The browser-based code appears to work similarly in Internet Explorer, FireFox, and Opera, but fails in Safari and Chrome. Note that the SEE Spacecraft Charging Handbook is a NASA code that is distributed by NASA.

Due to changes in Java security, the code requires the Java Control Panel security level be set to "Medium." However, starting with Java version 8u20 the "Medium" setting is no longer available. An alternative workaround (for today, at least) is to add the FILE protocol to the "Exception Site List," as shown in Figure 1. 


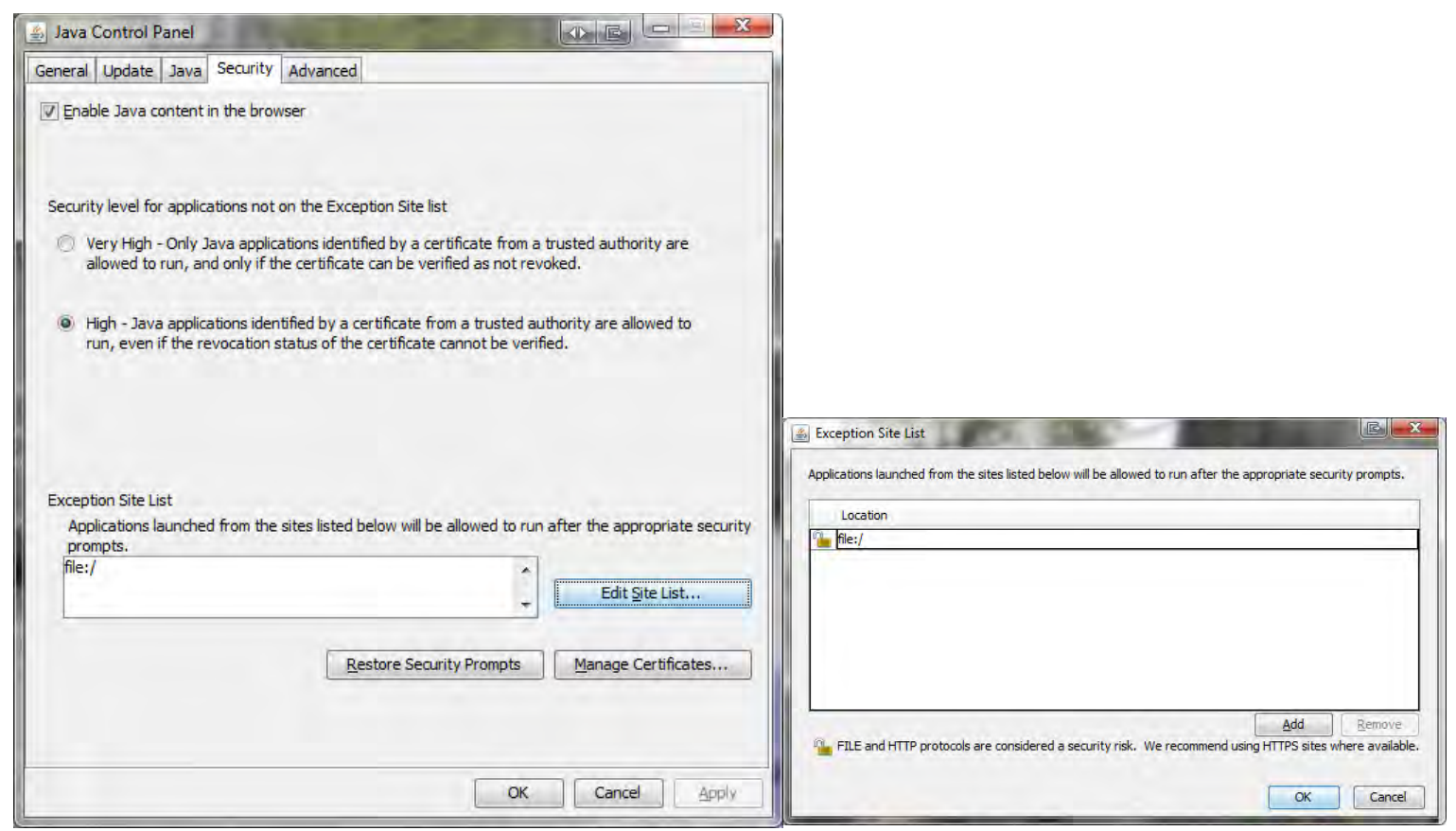

Figure 1. Adding the FILE protocol to the "Exception Site List."

\subsubsection{Charging in a LEO Plasma}

We explored the limitations and overlap between the various methods of modeling the charging of spacecraft with a low-Earth-orbit plasma within Nascap- $2 k$. This work resulted in a paper presented at the Spacecraft Charging Technology Conference in Pasadena, California. The paper included in the proceedings is reproduced below in Section 7.

\subsection{Reports and Meetings}

We participated in an in-person contract review at Kirtland AFB on May 14, 2014.

We attended the Spacecraft Charging Technology Conference in Pasadena, California, June 2327, 2014. We made an oral presentation titled Semi-analytic and PIC (Particle-in-cell) methods for Quantifying Charging in Dense, Cold Plasma. The paper is included in the conference proceedings and is reproduced in Section 7 below.

These presentations are included in the quarterly reports for the relevant periods.

\subsection{Scientific Reports and Journal Articles}

An oral presentation titled "Semi-analytic and PIC (Particle-in-cell) Methods for Quantifying Charging in Dense, Cold Plasma" was prepared and presented at the 13th Spacecraft Charging Technology Conference in Pasadena, CA in June 2014. The paper is included in the conference proceedings and is reproduced in Section 7 below. 


\subsection{Personnel}

Dr. Victoria A. Davis is the project manager and Dr. Myron J. Mandell is the principal investigator. The following Leidos staff members have contributed to the work reported here.

- Dr. Victoria A. Davis

- Dr. Myron J. Mandell

- Ms. Barbara M. Gardner

- Ms. Lisa Lattarulo

\section{REVISIONS TO NASCAP-2K SCRIPTING}

The Nascap-2k scripting was revised to eliminate obtuse commands. The changes were made backward compatible, so that old scripts still run correctly.

The object is now read from the XML file, placed in the database, and the surface potentials set to their initial values (from the XML file) by a new primary script command Read Object rather than by a subcommand of the Charge Surfaces script command. This dramatically reduces the complexity of scripts for calculations with fixed surface potentials.

The obscure subcommands of the Charge Surfaces command, "Calculate_Matrices", "DefineInsulators", "InitializeCalculation", "OpenDatabase", "PrepareChargeMatrix", "Read_Matrices", "ReadObject", "ReadPotentials", and "WritePotentials", were eliminated. Initialization and database access operations are now performed automatically as needed.

The subcommands used to set calculational parameters, "Compute_Transverse_Currents", "FieldsFromFile", "SpaceChargeLimitedPhotoemission", "ZeroCurDerivAlgorithm", and "ZeroTotCurAlgorithm" were combined into a single "Set Parameters" subcommand.

Scripts for common calculations are now simpler. Figure 2 through Figure 4 show the more compact scripts for some common calculations. 


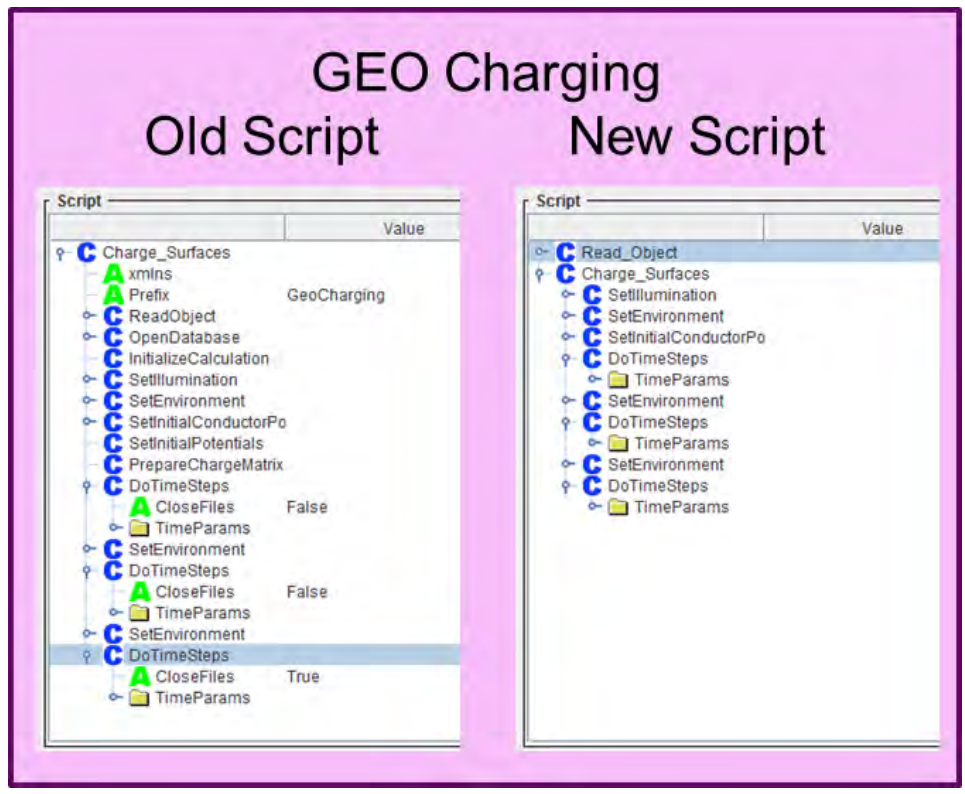

Figure 2. Comparison of Scripts Used to Compute Charging in a Geosynchronous Substorm using the Old and New Schemes

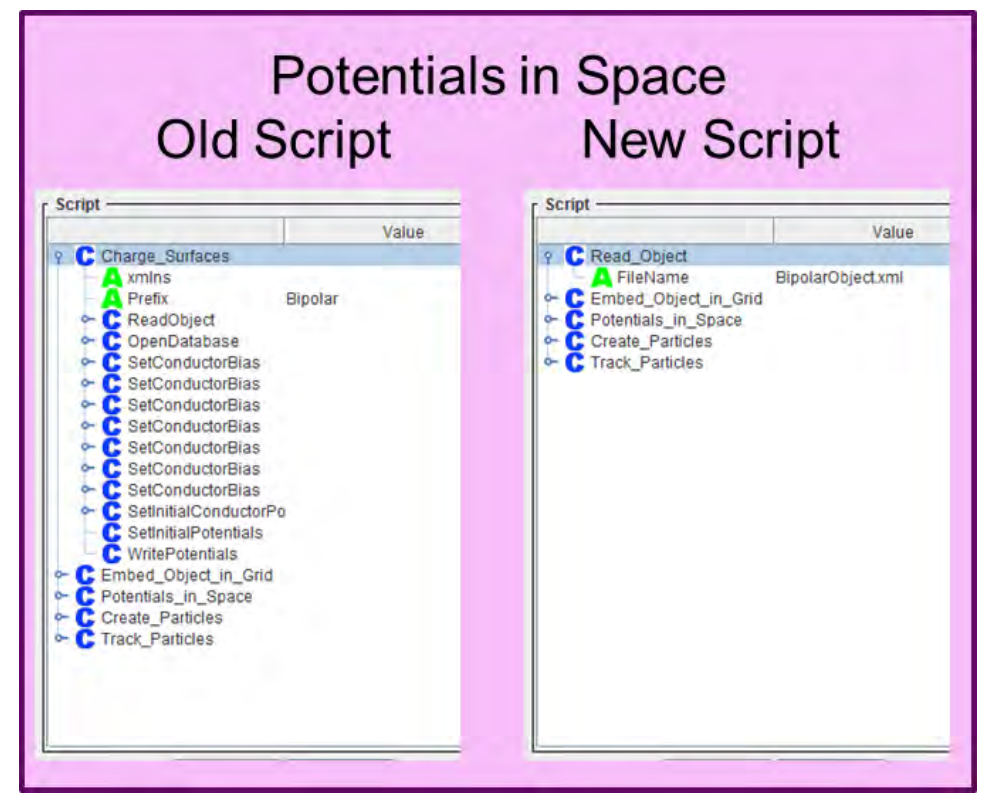

Figure 3. Comparison of Scripts Used to Compute Potentials in Space Given Specified Surface Potentials Using the Old and New Schemes 


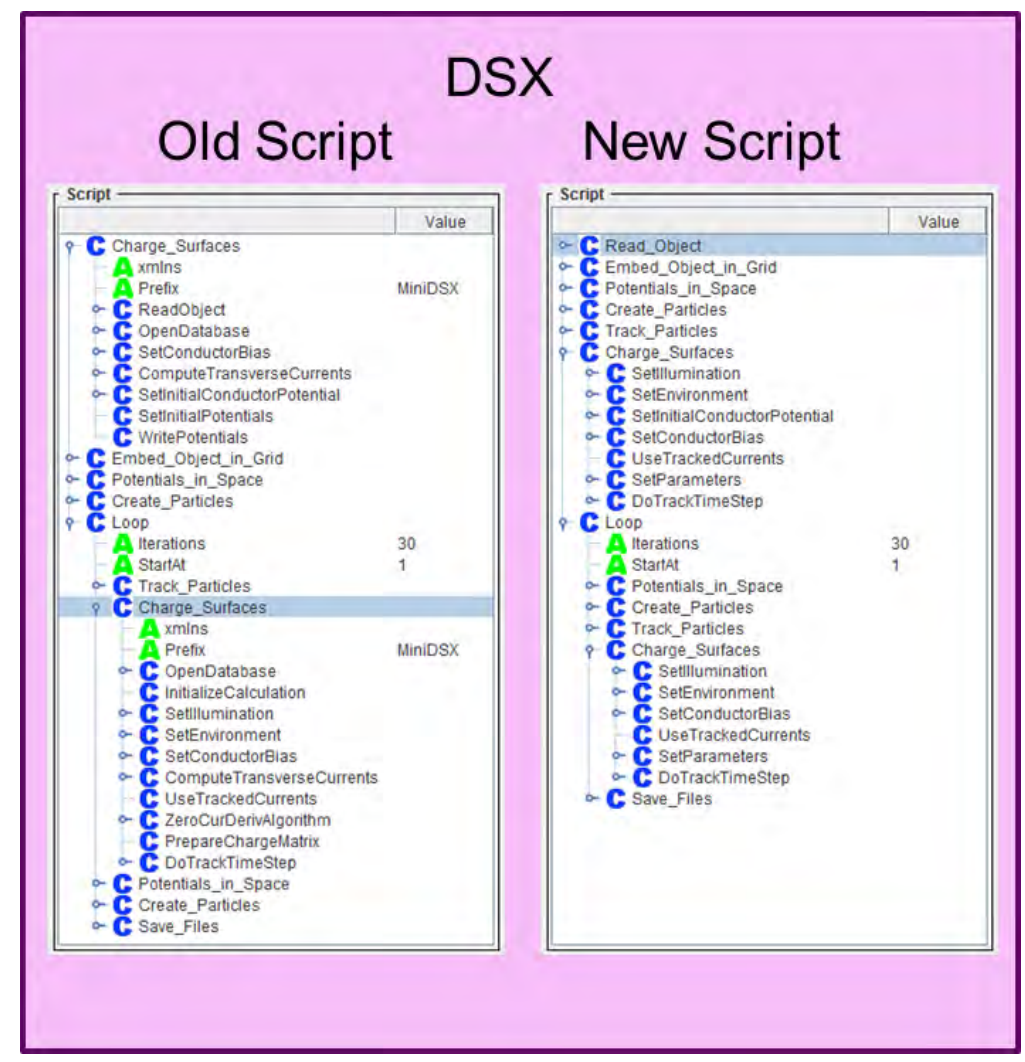

Figure 4. Comparison of Scripts Used to Specify the DSX Charging Problem Using the Old and New Schemes

Nascap- $2 k$ standard test problems were used to verify that the scripting changes are adequate and that they were correctly implemented. The suite of standard test problems was expanded to include at least one computation that uses each of the available script commands. Using the existing scripts, the standard test problems were executed using the code from before the changes and using the code from after the changes. All the differences in the text output files were examined and the causes determined. All issues were fixed.

For the test problems that use a default script, a new default script was generated. For test problems that use a modified script, the script was recreated using the new formulation. The calculations were done using the new script and all differences in text output examined and the causes determined. All issues were fixed.

\section{GENERALIZATION OF NASCAP-2K CHARGING CAPABILITIES}

Nascap-2 $k$ was modified to allow for a wider variety of charging calculations to be easily specified. Table 1 lists the charging calculations that can now be specified within the user interface. 
Table 1. Charging Calculations Allowed by Default (Blue Text Indicates New Options)

Geosynchronous

- Analytic currents (Maxwellian, Double Maxwellian, Kappa, Tabular)

- No velocity, Only H+ ions

LEO or Plume

- Analytic currents (Convected Maxwellian for user specified ion species and for electrons)

- Tracked particle currents

- Tracked ion and analytic electron currents (Convected Maxwellian)

Auroral

- Analytic currents (Convected Maxwellian for user specified ions species and Fontheim for electrons)

- Tracked ion and analytic electron currents (Fontheim)

Interplanetary

- Analytic currents (Convected Maxwellian for user specified ions species and for electrons)

- Tracked ion and analytic electron currents (Convected Maxwellian)

LEO/Time dependent plasma

- Tracked particle currents

- Tracked ion and analytic electron currents (Convected Maxwellian)

The user interface was modified in two ways. The code that enables and disables the options available on the Problem tab was modified to allow for the wider variety of calculations to be specified. Also, the code that writes the default scripts was modified to allow for the additional calculations. At the same time the default script code was streamlined.

The most important changes made are those in the code that computes the current to a surface and its derivative. The code was both modified and cleaned up. The tight coupling between the type of environment and the current calculation was reduced. The code that computes the current and the code that computes its derivative were reorganized to make their structure clearer and extensive comments were added.

Analytic formulas for the derivative of the current with respect to the potential for the Fontheim and Convected Maxwellian distributions were computed. For the Fontheim distribution the formula is exact, and for the Convected Maxwellian it is approximate. These formulas were implemented in the code to replace the numeric derivatives previously used.

The capability to compute and use secondary electron currents in all charging calculations with tracked currents was added. The yield is computed using analytic currents with the correct ion masses.

The current to surfaces was computed in four separate methods (subroutines). The code was reorganized so that all four methods call a single method containing the current computation code. 
Sample calculations for charging (current balance) in a cold, dense plasma indicated that the last step in the computation of the derivative of the current with respect to the potential needed to be changed. Previously, the derivative was always set to be large enough so that the estimated change in potential during the next timestep is at least $1 \mathrm{~V}$. For stability, the minimum estimated change in potential needed to be reduced.

The following example was used to test the new capabilities: charging in a cold, dense plasma using currents computed for a convected Maxwellian plasma. The Object Toolkit model was constructed at AFRL to model a LEO spacecraft. The geometry is shown in Figure 5, with the results in Figure 6. The solar array is divided into four sections with different potentials from chassis plus $50 \mathrm{~V}$ to chassis plus $200 \mathrm{~V}$. The potentials on the gold surfaces reflect these values. The results are as expected. The chassis potential rapidly decreases to $-140 \mathrm{~V}$. The insulating OSR and solar cell surfaces show immediate potential swings with the underlying conductors. The insulating surfaces then differentially charge to small potentials reflective of the plasma temperature.

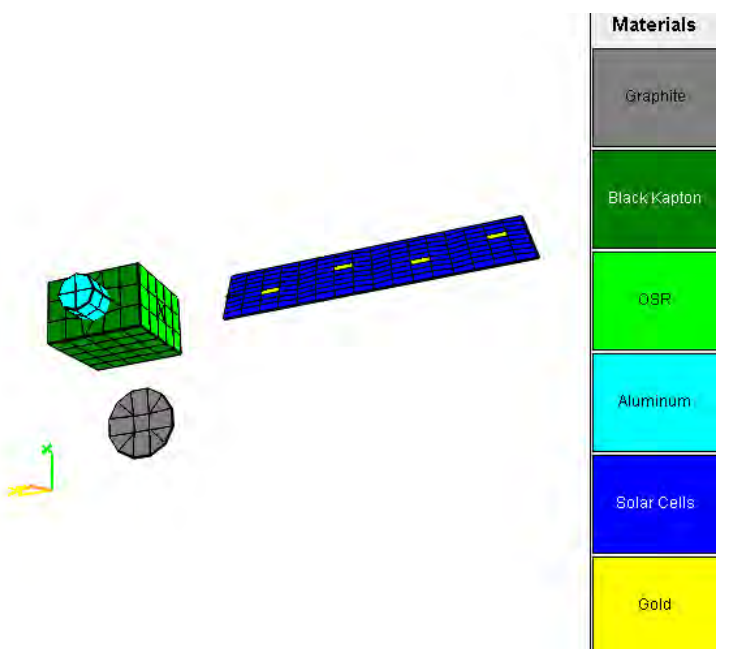

Figure 5. Geometry and Materials for First Test Case for Charging in LEO with Analytic Currents from a Convected Maxwellian Distribution 

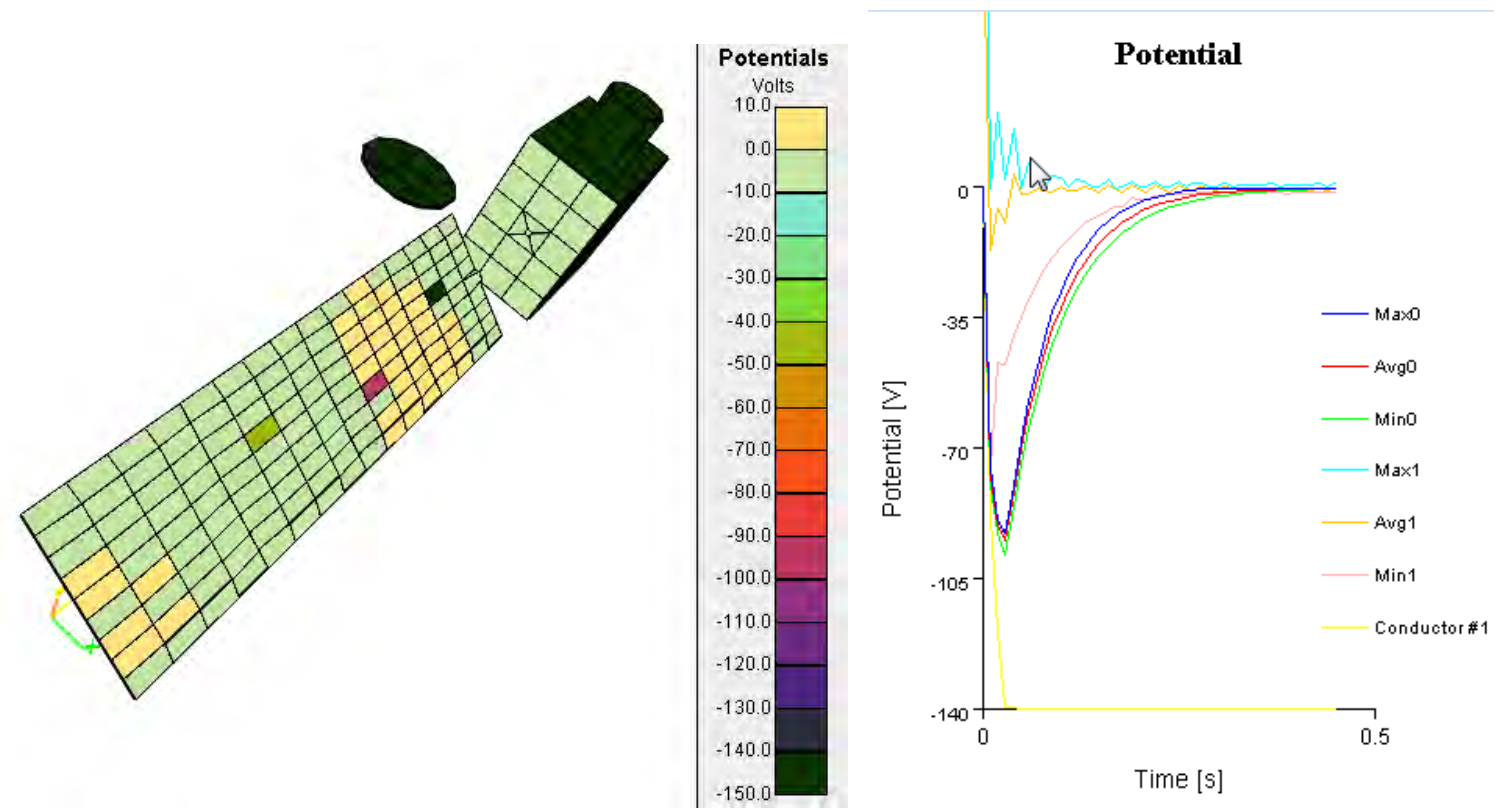

Figure 6. Potentials Resulting from Charging Calculation using Geometry of Figure 5

\section{COMPREHENSIVE TESTING}

Comprehensive testing of the new code was performed.

Calculations done earlier (both the standard test problems and others) were repeated with the new code and either the old or regenerated scripts. To speed up these comparisons, flags were modified to reduce non-meaningful differences in text files (such as the time since execution started). Also, Nascap- $2 k$ was modified so that random number generation can occur with a fixed seed or with a random seed. Test problems that use the random number generator were adjusted to use a fixed seed.

Also, calculations enabled by recent code enhancements were performed and the results compared with expected values.

Also, the standard suite of test problems was executed on LINUX and the appropriate comparisons done.

Examples of this testing follow.

\subsection{Big Grid Test}

A "Potentials in Space" calculation was done with a grid with a large number of volume elements. The computational grid is $121 \times 101 \times 51$ with mesh spacing of $6.7 \mathrm{~cm}$. The potentials about a $-10 \mathrm{~V}$ parallelepiped in a $4 \mathrm{~cm}$ Debye length plasma were computed. The results are shown in Figure 7. 


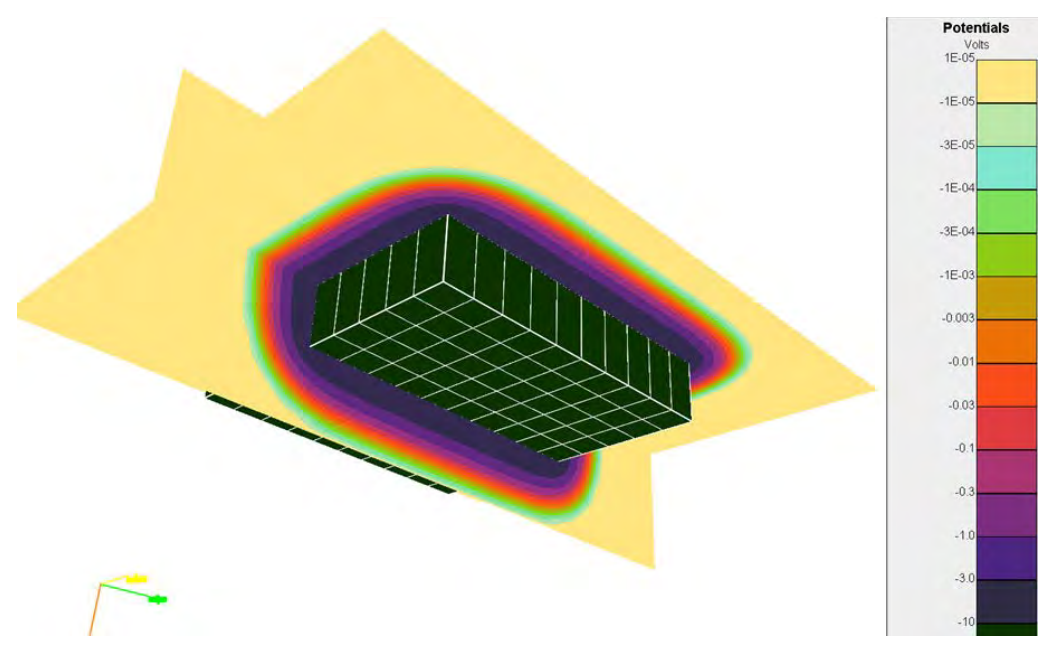

Figure 7. Potentials about -10 V Parallelepiped in a 4 cm Debye Length Plasma

\subsection{Magnetic Dipole}

Verified that the capability to track particles in a dipolar magnetic field still functions correctly by tracking electrons from the edge of a sheath to a $+10 \mathrm{~V}, 1 \mathrm{~m}$ radius sphere with a $300 \mathrm{Am}^{2}$ magnetic dipole at its center. The potentials and the trajectories are shown in Figure 8.
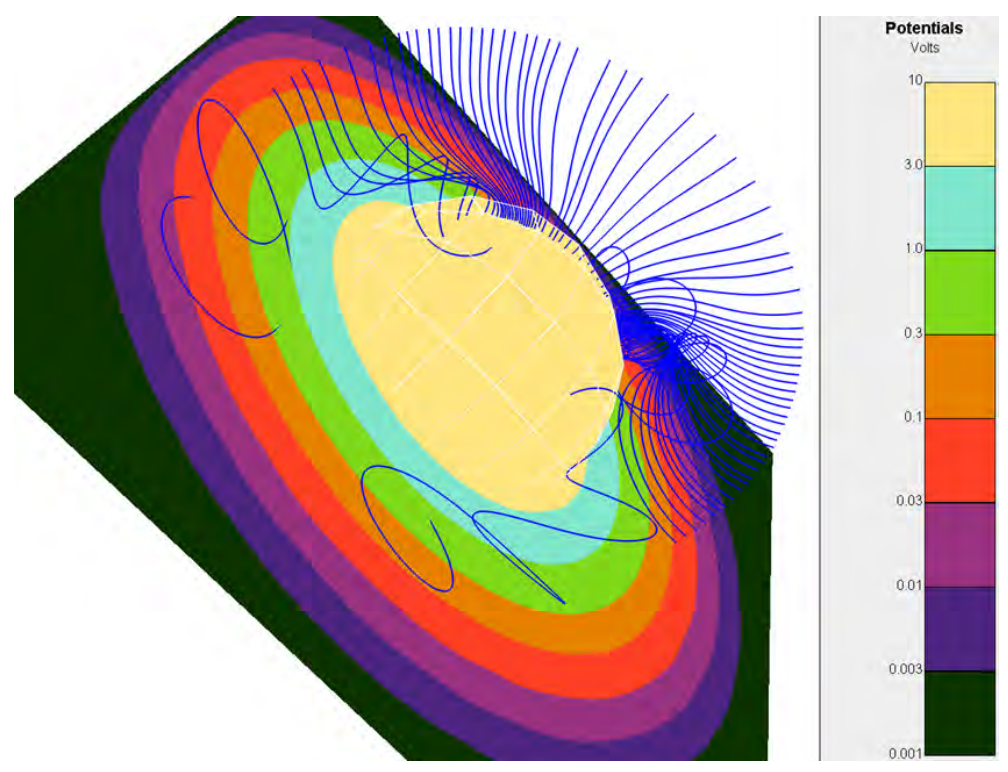

Figure 8. Electron Trajectories toward a +10 V Sphere with a Magnetic Dipole at Its Center (Potentials in a Plane through the Center of the Sphere Are Also Shown)

\subsection{Simple $v \times B$ Test}

An earlier calculation done while developing the capability to include $\mathbf{v} \times \mathbf{B}$ potentials in charging calculations was repeated to verify that these potentials are still included correctly after the recent changes of the scripting and charging coding. The existing (previously developed) script (shown in Figure 9) was used. The geometry used is a metal cylinder with Teflon collars as 
shown in Figure 10. The magnetic field is taken to be 7.1 gauss, and the velocity is taken to be $600 \mathrm{~km} / \mathrm{s}$, for an induced potential of $1700 \mathrm{~V}$ along the $4 \mathrm{~m}$ long cylinder. The plasma density starts at $10^{6} \mathrm{~m}^{-3}$ and increases by one order of magnitude each second to $10^{10} \mathrm{~m}^{-3}$ from 4 seconds to the end of the calculation at 10 seconds. The electron and ion temperatures are both taken as 1 eV. A constant timestep of 0.2 seconds was used.

The results shown in Figure 11 through Figure 13 are as expected. The $\mathbf{v} \times \mathbf{B}$ potentials appear on the exposed conductor surface elements. The insulators are initially at the same potential as the underlying conductor (zero differential potential) and charge to zero absolute potential, at first slowly and then more rapidly as the plasma density is increased, as shown in Figure 12. The charging current to insulators at the negative end shows roughly an order of magnitude increase each time the plasma density is increased, while the charging current to insulators at the positive end remains small, as shown in Figure 13.

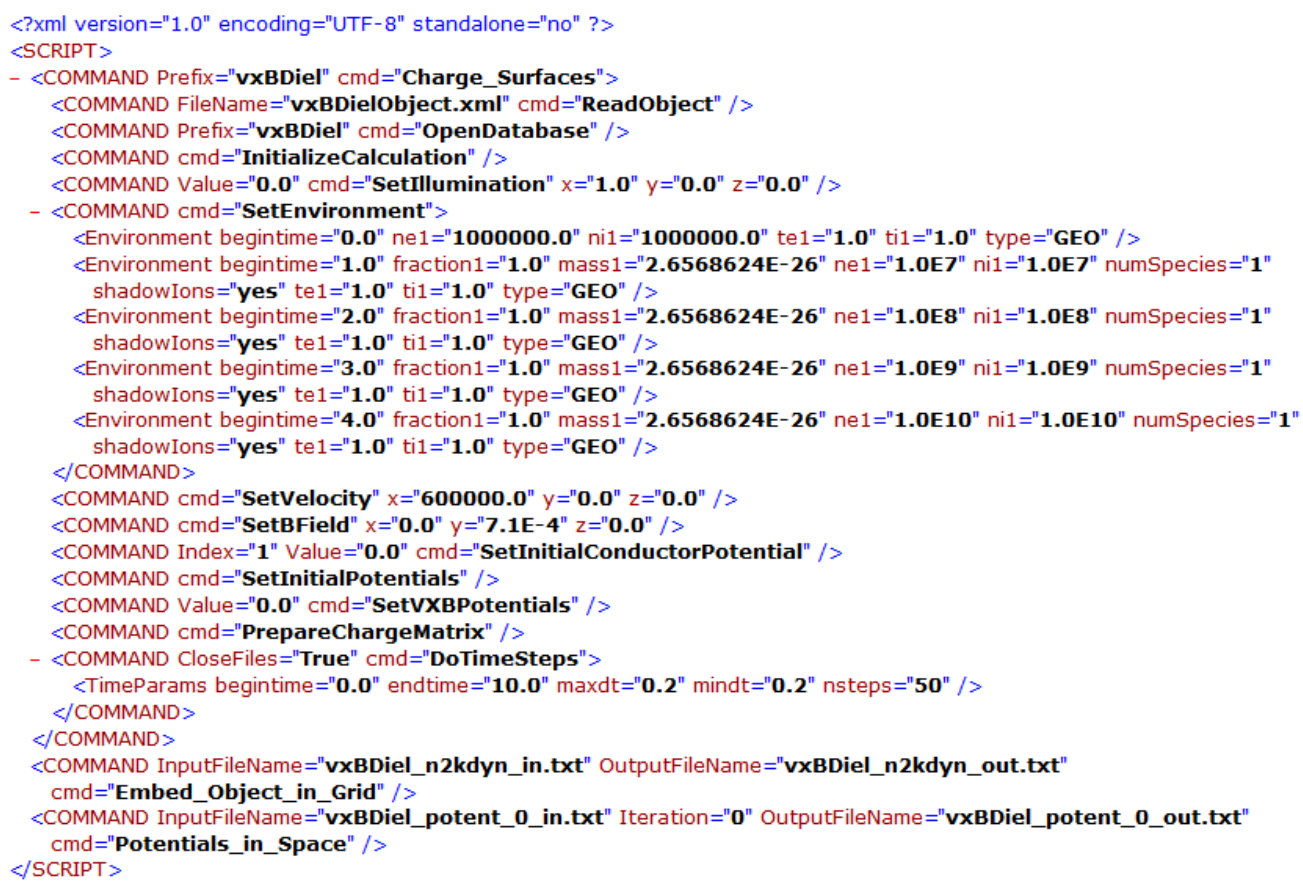

Figure 9. Script Used for “Simple $v \times B$ ” Test 


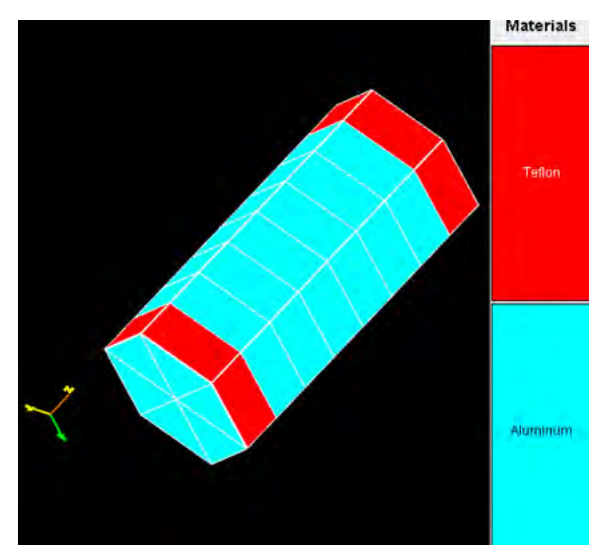

Figure 10. Object Toolkit Object Used in Verification Calculation
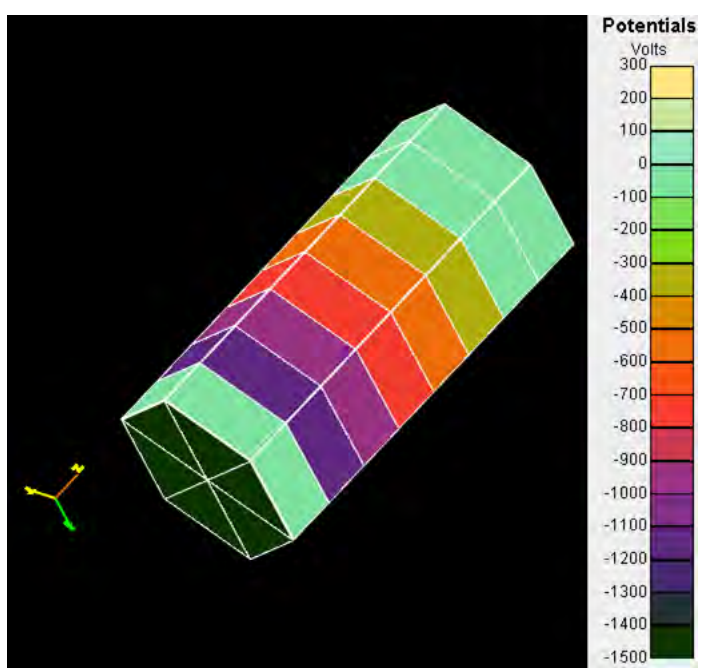

Figure 11. Final (Steady State) Surface Potentials for the Verification Calculation 
Potential

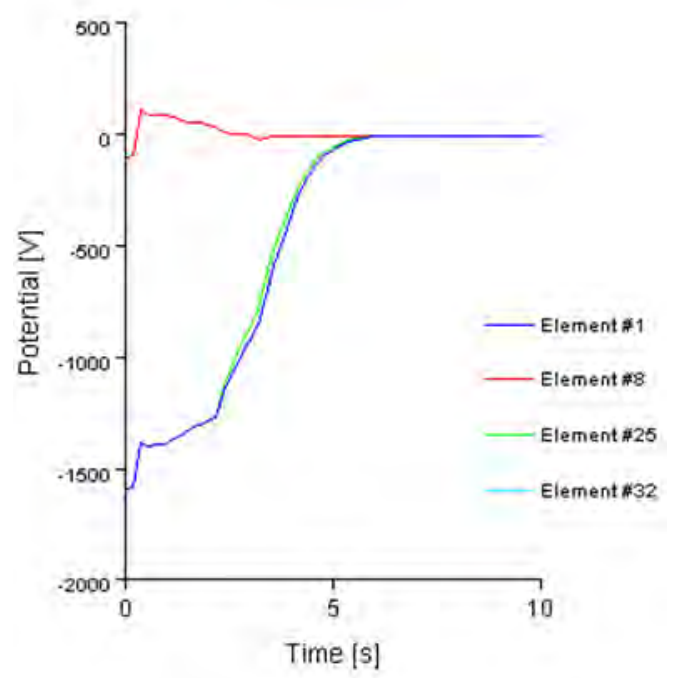

Differential Potential

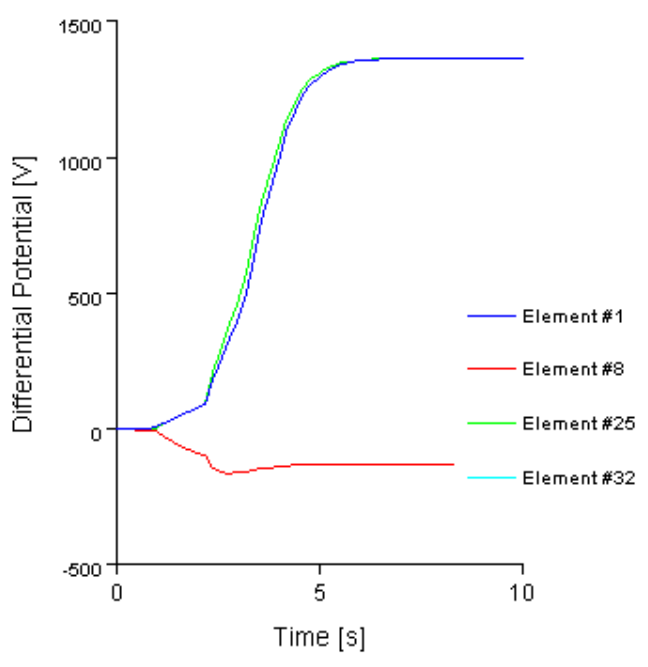

Figure 12. Absolute and Differential Potential as a Function of Time (and Density) at Selected Insulating Surface Elements (Two Surfaces from Each Insulating Collar are Shown)

\section{Charging Current}

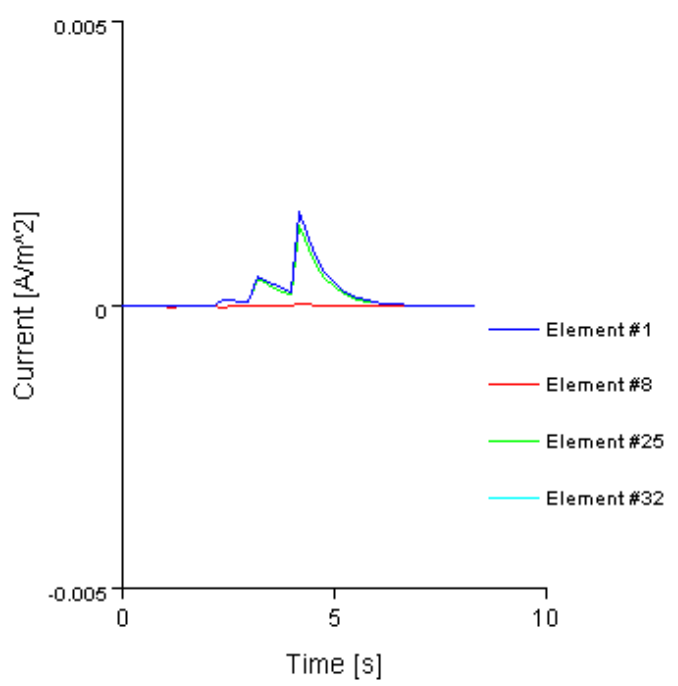

Figure 13. Charging Current to Insulating Elements at Each End of the Cylinder as a Function of Time (and Density)

\subsection{Juno}

An earlier calculation of the potentials expected on the Juno spacecraft during a polar pass over the Jupiter pole for specified conditions was repeated to verify that the capabilities used for that 
calculation still work correctly. The calculation tests the ability to simulate a spinning satellite moving rapidly through a high magnetic field, time-dependent environment. In particular, we wanted to be sure that the differential potentials are preserved across successive Charge Surfaces commands, and that the time-dependent environment was updating properly,

The previously generated script was used, with the exception that "starttime" keyword for the environment were changed to "begintime" keywords to match the new convention. The spacecraft rotates at $5 \mathrm{rpm}$. The velocity vector $(60 \mathrm{~km} / \mathrm{s})$ lies in the plane of rotation, along with a 1 gauss magnetic field perpendicular to the velocity. The magnetic field normal to the plane of rotation was 10 gauss, giving a $\mathbf{v} \times \mathbf{B}$ field in the plane of rotation of $60 \mathrm{~V} / \mathrm{m}$. Surface potentials on the bottom (conductive) side and on the top (solar cell) side are shown in Figure 14.

The code correctly executed the test case. It was noted that the first environment in the first set is automatically reset to have a "begintime" of 0.0 .
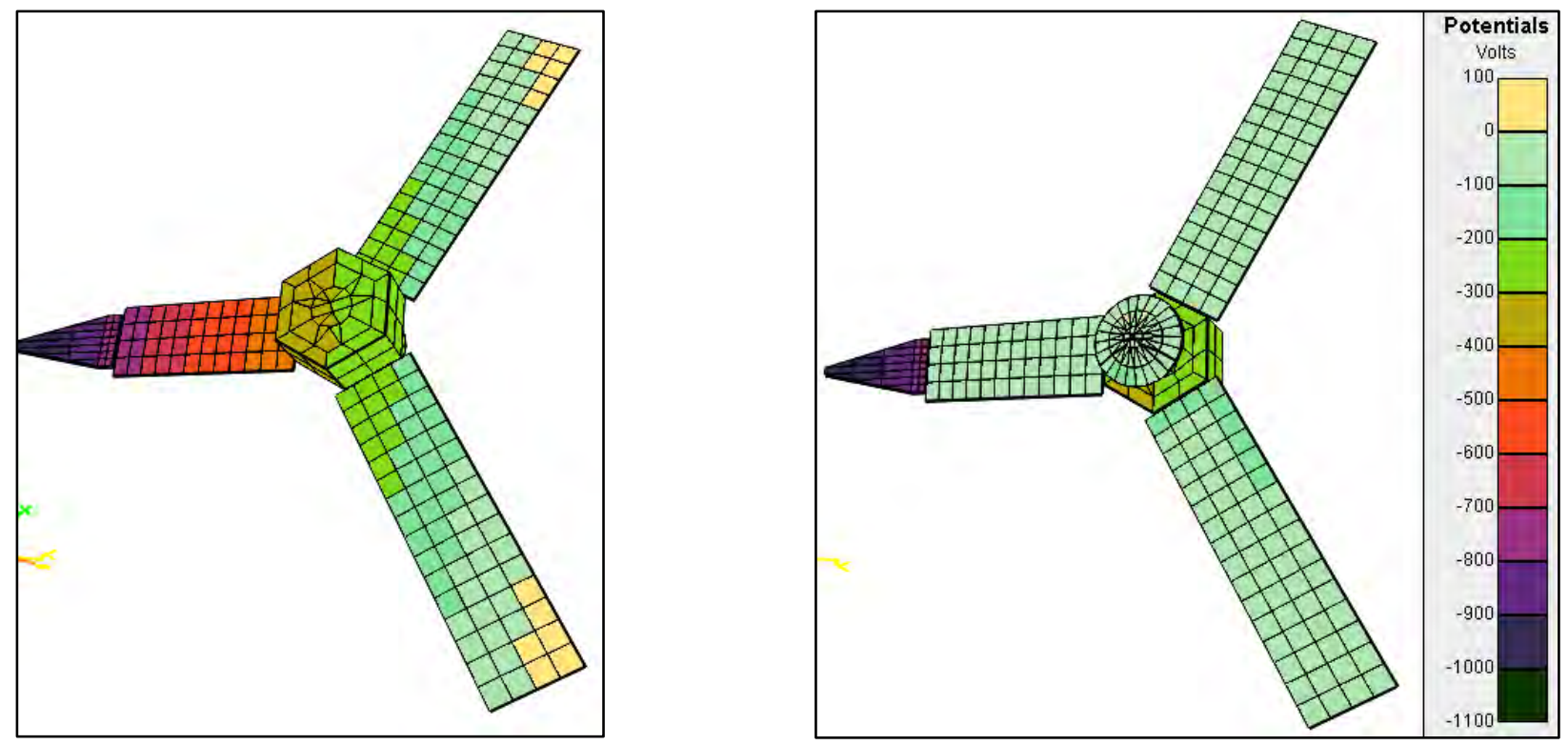

Figure 14. Surface Potentials on Juno for the Bottom (Conductive) Side (Left) and the Top (Insulating Solar Cell) Side (Right).

\subsection{SPEAR 3}

Calculation of the potentials about the SPEAR III rocket and its magnetically limited current collection was performed. This calculation had not been done since about 1995. The Patran model of SPEAR III was imported into Object Toolkit and materials and conductor numbers assigned to surfaces. The density was set to $10^{11} \mathrm{~m}^{-3}$, the temperature to $0.3 \mathrm{eV}$, and the ion species to Oxygen. Bias of $10 \mathrm{kV}$ was applied to the sphere, with $750 \mathrm{~V}$ increments on the graded boom, and the body was set to $-3 \mathrm{kV}$. Potentials were calculated using the "Non-linear" formulation, giving the results shown in Figure 15. Then particles were tracked with no magnetic field and with 0.3 gauss along the boom or normal to the plane of the model. The resulting currents, positive to the body and negative to the boom and sphere, are shown in Table 2 . They follow the expected pattern. 

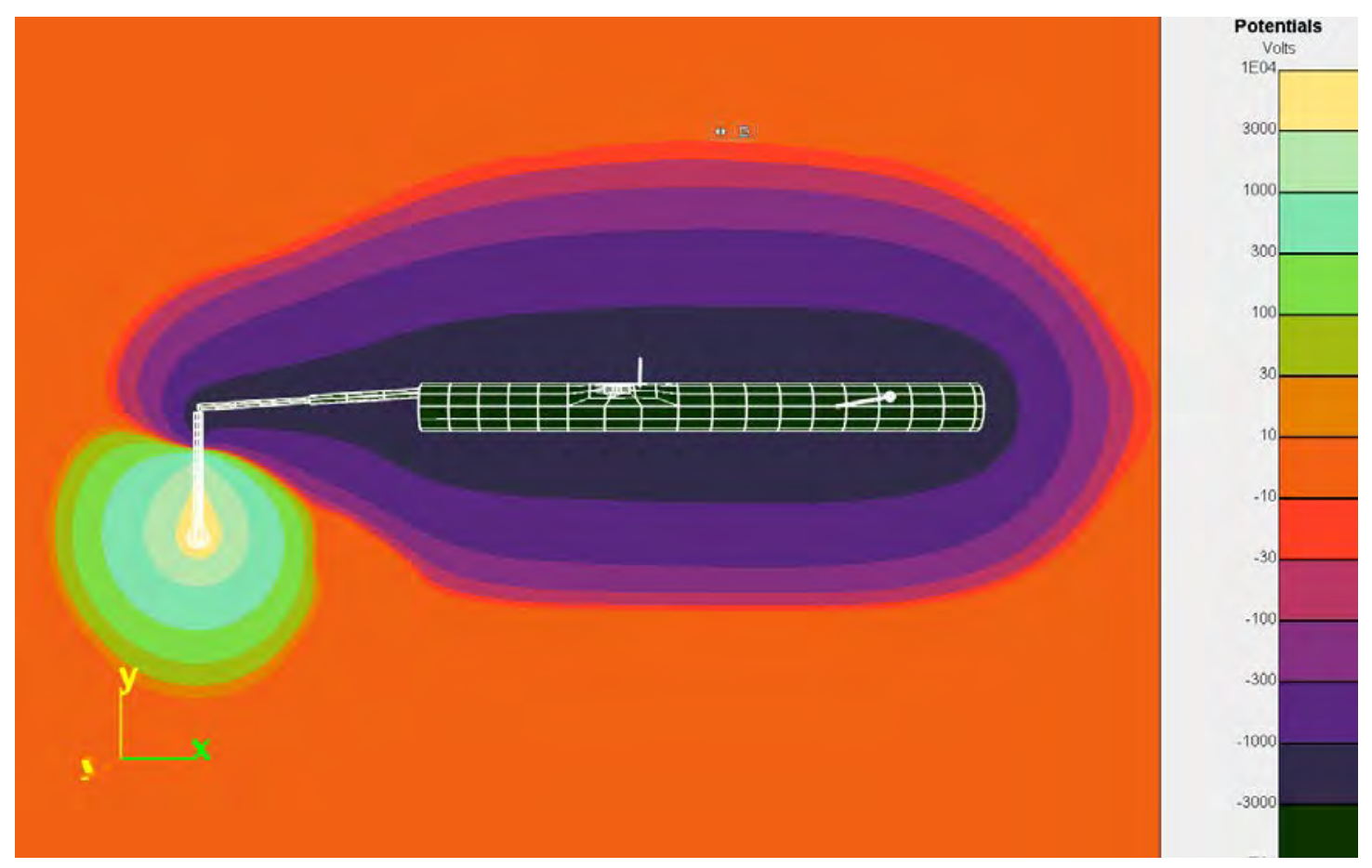

Figure 15. Potentials about the SPEAR III Model, with Parameters as Given in Text

Table 2. Calculated Currents to the SPEAR III Model for Parameters Given in Text and for Three Magnetic Field Configurations

\begin{tabular}{|c|c|c|c|c|c|c|}
\hline $\begin{array}{c}\text { Chassis } \\
\text { potential }\end{array}$ & $\begin{array}{c}\text { Sphere } \\
\text { potentials }\end{array}$ & $\begin{array}{c}\text { Magnetic field } \\
\text { (gauss) }\end{array}$ & $\begin{array}{c}\text { Current to } \\
\text { body (A) }\end{array}$ & $\begin{array}{c}\text { Current to } \\
\text { sphere (A) }\end{array}$ & $\begin{array}{c}\text { Current to } \\
\text { boom (A) }\end{array}$ & $\begin{array}{c}\text { Total } \\
\text { current (A) }\end{array}$ \\
\hline-3000 & 7000 & $(0,0,0)$ & 0.00103 & -0.0306 & 0 & -0.030 \\
\hline-3000 & 7000 & $(0,0.3,0)$ & 0.00103 & -0.0108 & -0.00032 & -0.010 \\
\hline-3000 & 7000 & $(0,0,0.3)$ & 0.00103 & -0.0085 & -0.00837 & -0.016 \\
\hline
\end{tabular}

\subsection{MMS}

Three earlier calculations of the charging of the MMS spacecraft were repeated to verify that a standard long Debye length charging calculation is still easily defined and that the results remain as earlier. The three calculations have different environments and timesteps. All three were executed with the old script and with a rebuilt default script. The results are identical within expected numeric variation.

\subsection{Solar Probe Plus}

An earlier calculation of the charging of the Solar Probe spacecraft was repeated to verify that the functionality used to compute charging in the Solar Wind continues to work correctly. Once the parameters were adjusted to be identical to those used previously, the same results were obtained, within the expected numeric variation. 


\subsection{Emitter Test Case}

In order to explore and test Nascap-2k's ability to model an emitter, the floating potential of a cylinder with an emitter on its side was computed. The current balance is between the emitter electron current and sheath electrons collected in Parker-Murphy fashion from a LEO plasma.

A simplified version of this test case has been added to the Nascap-2k User's Manual as an example.

\subsubsection{The Parameters}

The object is a $1 \mathrm{~m}$ long, $0.4 \mathrm{~m}$ diameter, aluminum cylinder, as shown in Figure 16. A current of $0.3 \mathrm{Am}^{-2}$ of electrons is emitted from a surface with area $0.03451 \mathrm{~m}^{-2}$, for a total current of $10.35 \mathrm{~mA}$. The environment parameters are set as shown in Figure 17. The magnetic field is parallel to the cylinder axis, and an extra species is defined for emitted electrons. On the Problem tab the environment is specified as "LEO or Plume" and the problem type is specified as "Charging" with "Tracked Particle Currents". The conductor is specified as floating with an initial potential of $+5 \mathrm{~V}$. The computational space is three nested grids with a $20 \mathrm{~cm}$ resolution outer grid and a $5 \mathrm{~cm}$ resolution inner grid. The emitter properties are shown in Figure 18.

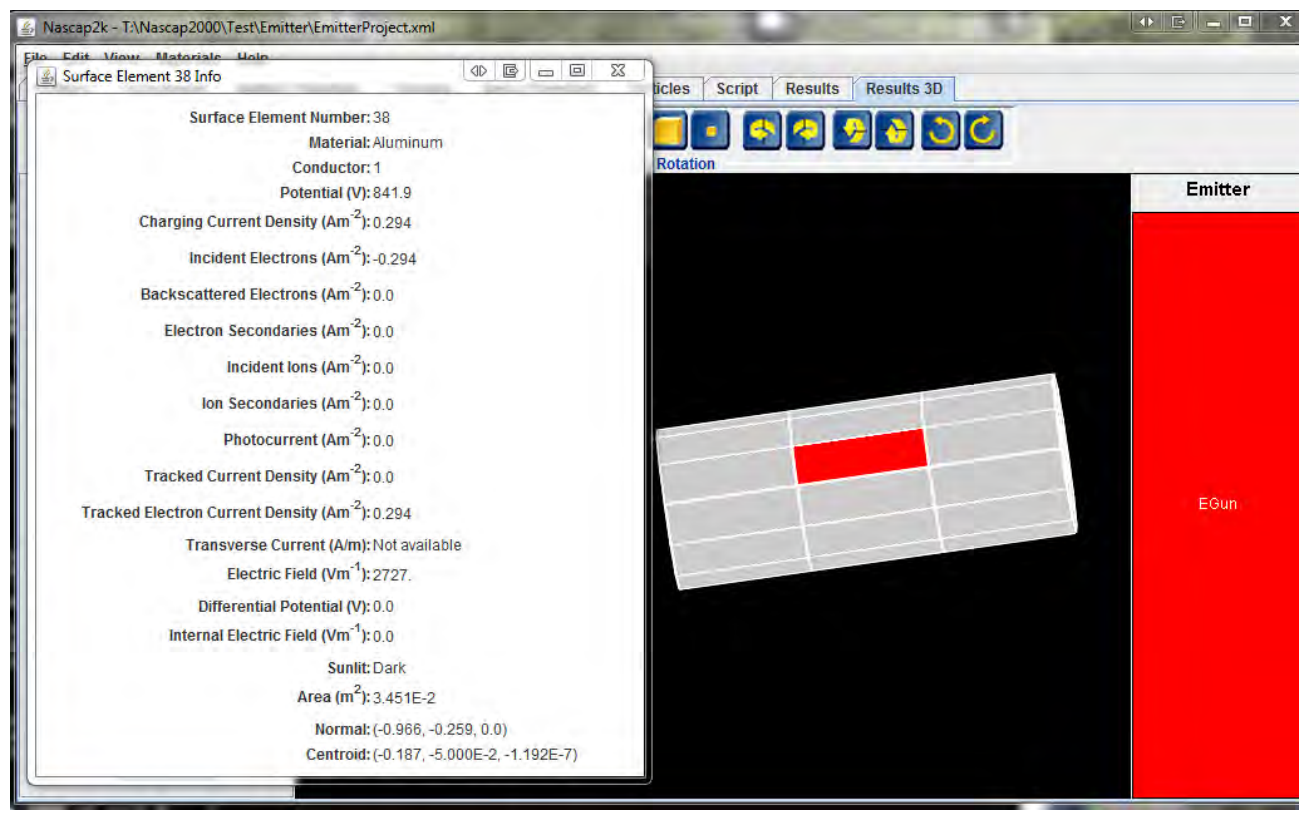

Figure 16. Aluminum Cylinder Showing Emitter Surface Element 


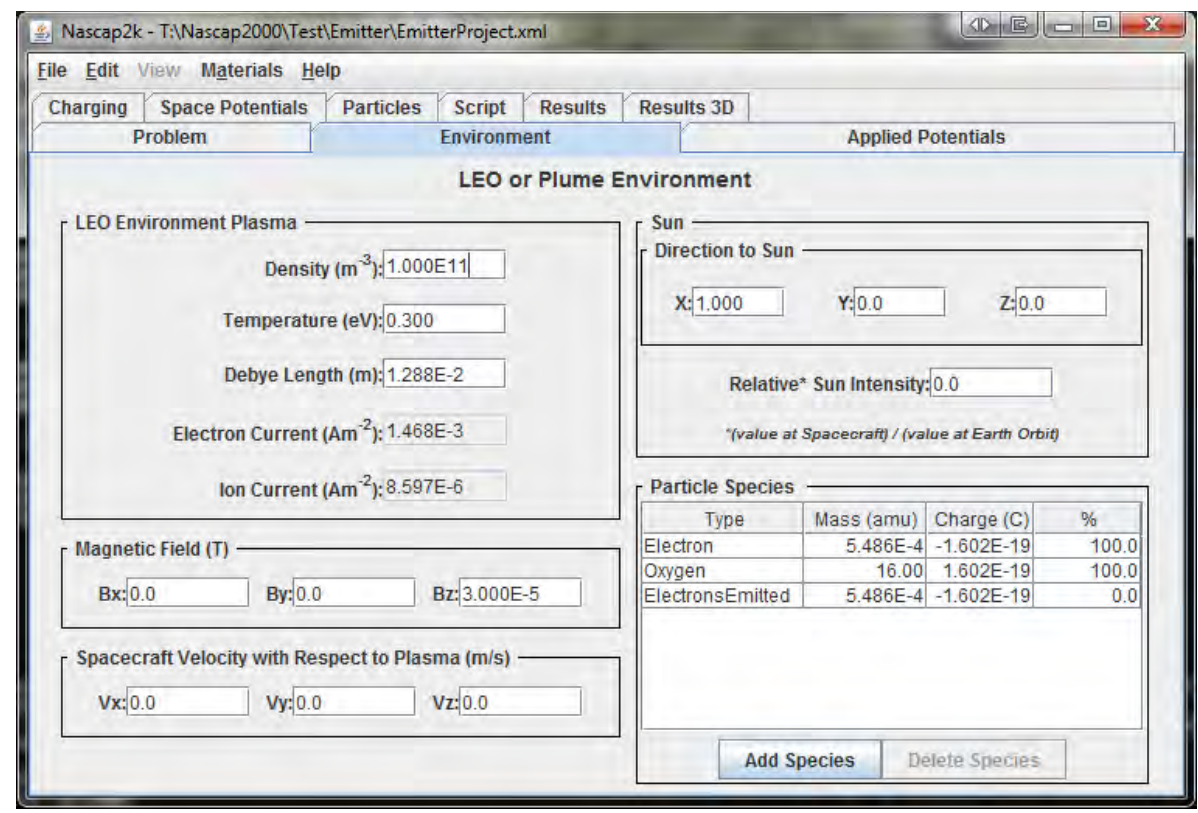

Figure 17. Environment Parameters

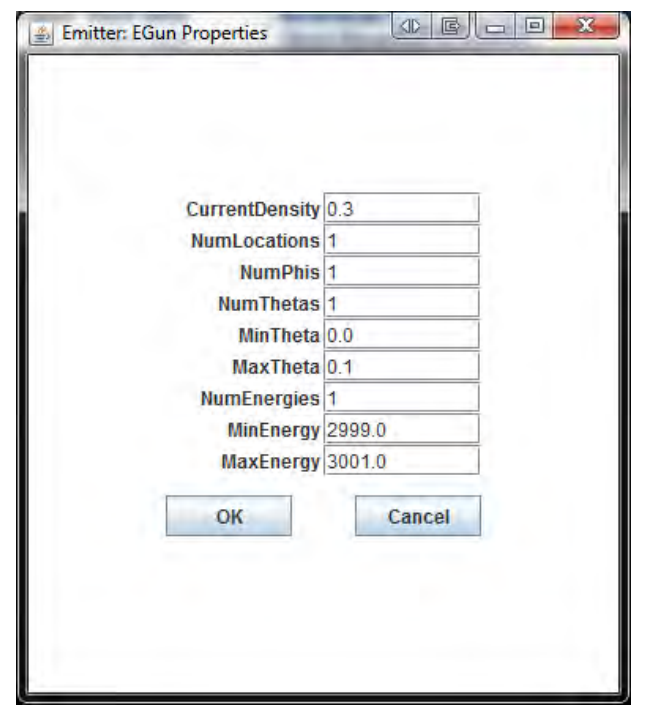

Figure 18. Emitter Parameters

\subsubsection{Building the Script}

The script for this problem is nonstandard. The following procedure was used.

1. On the Charging tab, set $200.1 \mathrm{~ms}$ timesteps.

2. On the Particles tab, make the appropriate selections for the emitter electrons, as shown in Figure 19.

3. On the Script tab, build the script. Make sure the "Automatically overwrite files" box is unchecked. 
4. For all the Create Particles and Track Particles commands, set the index of the input file to 0, as shown in Figure 20.

5. Click "Save Files." Click "Yes to All" on the overwrite question.

6. On the Particles tab, make the appropriate selections for the sheath electrons, as shown in Figure 21.

7. Return to the Script tab and rebuild the script.

8. Make the same edit specified in item 4 above.

9. Save the files with overwrite, as in item 5 above.

10. Duplicate each of the three Create_Particles commands, as shown in Figure 22.

11. In one of each pair of Create_Particles commands, change "Electron" to "ElectronsEmitted" (three places) as shown in Figure 23. (The Track_mode, Creation_mode, and Species values are used to write the input file. When existing input files are used (as in step 13 below) the values are ignored.)

12. Edit the Tracker input file to specify both species, as shown in Figure 24.

13. Run the script, this time answering "No to All" on the overwrite question.

Note that several methods are available to construct the desired input files and script.

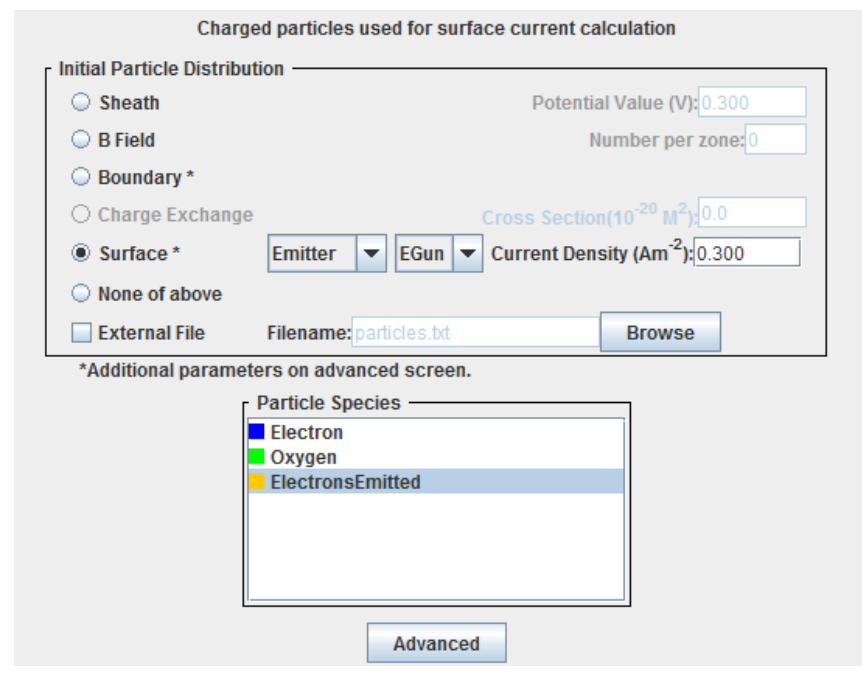

Figure 19. Particles Tab Specification for Emitter Electrons 


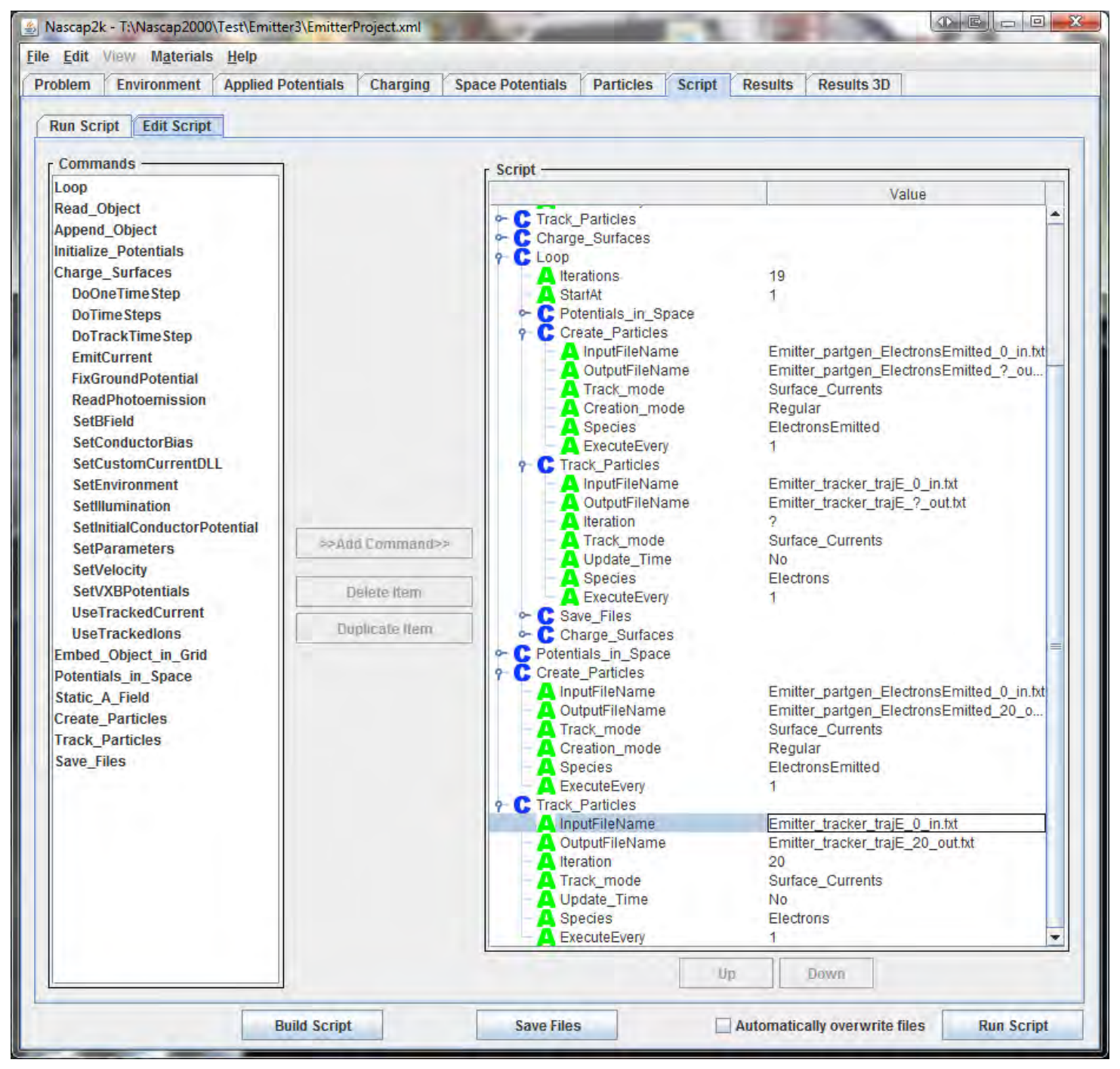

Figure 20. Editing of Script to Simplify Electron Emitter Files 


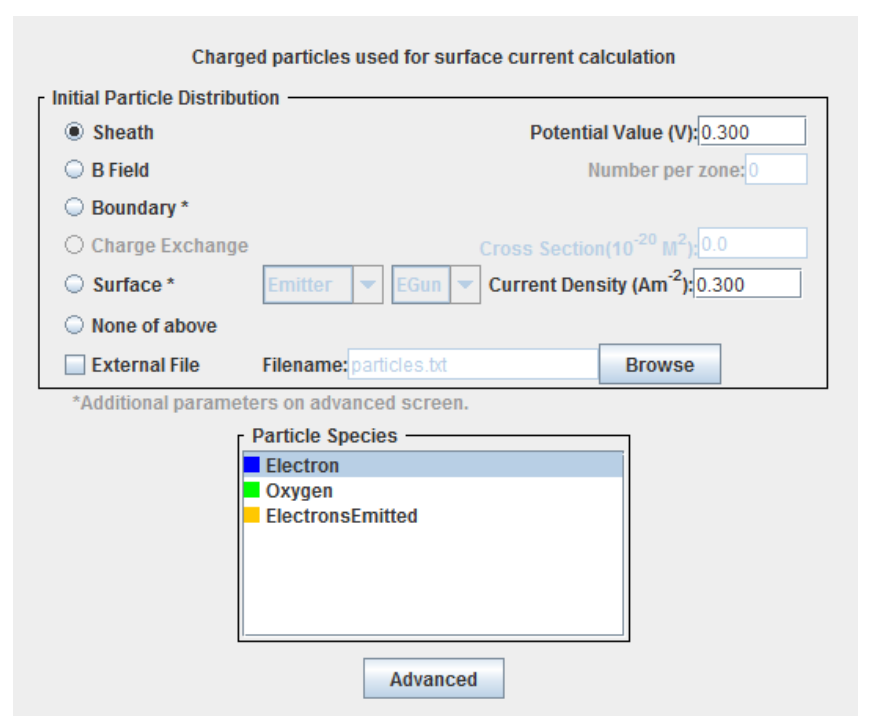

Figure 21. Particles Tab Specification for Sheath Electrons

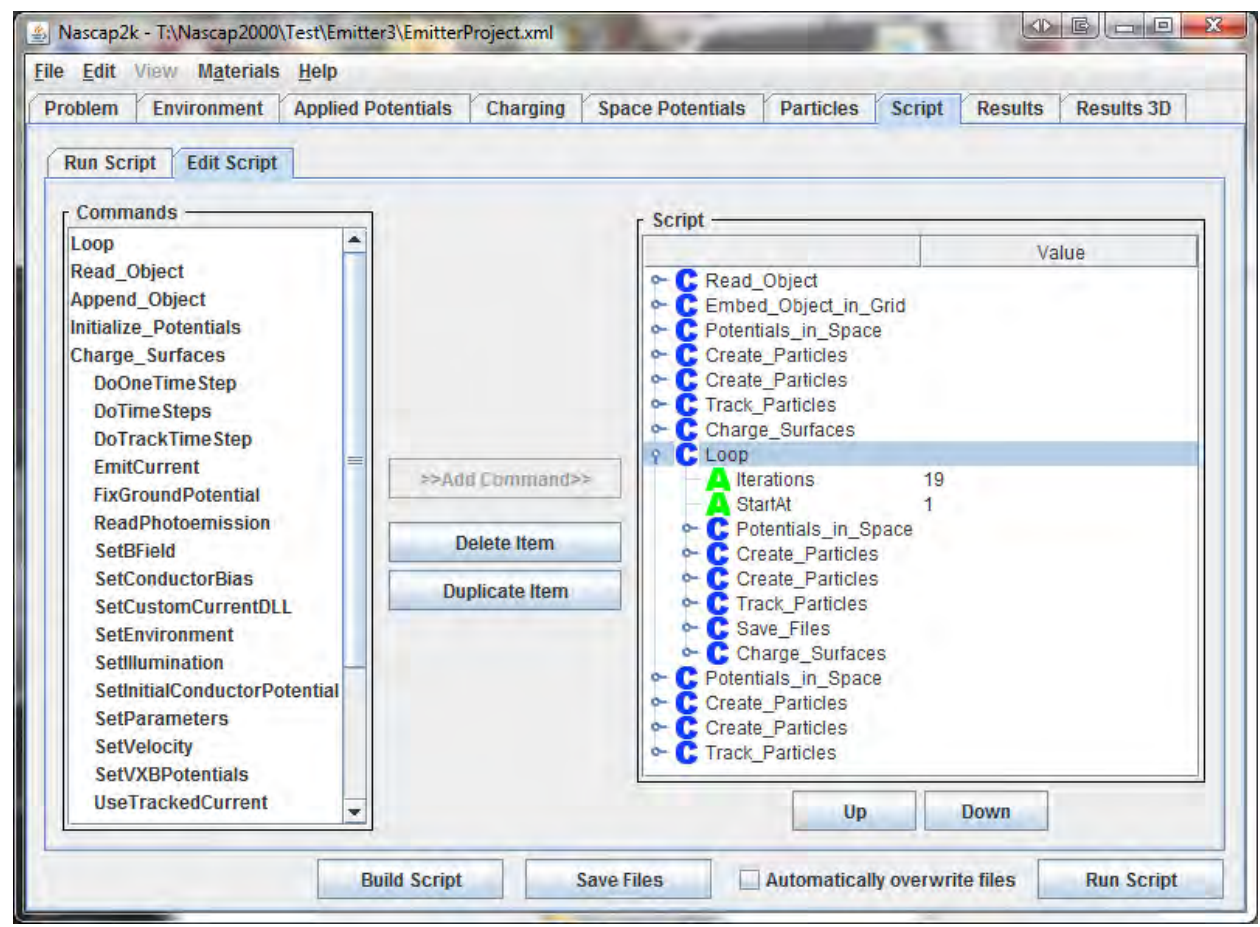

Figure 22. Script with Duplicated Create Particles Commands 


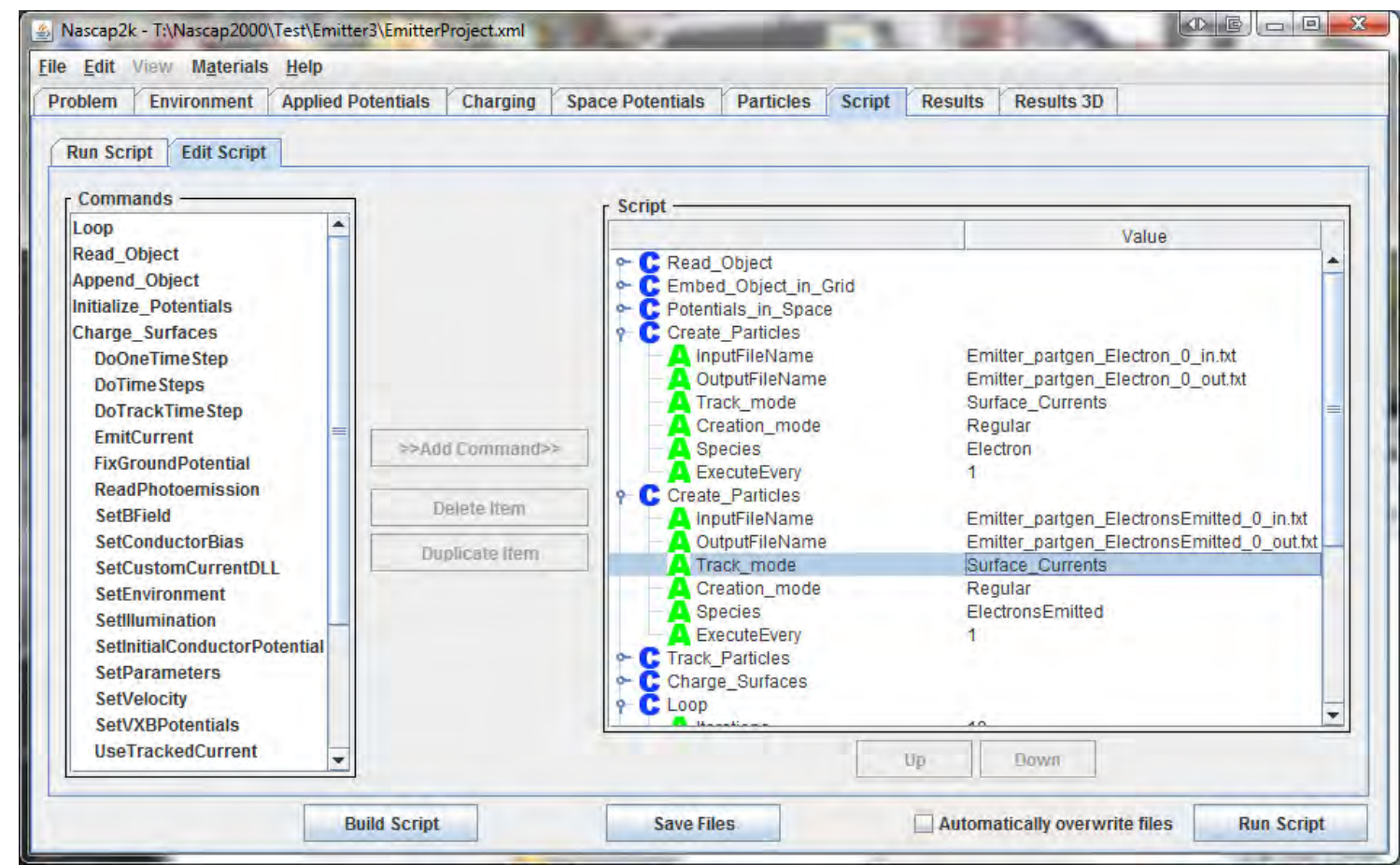

Figure 23. Editing of a Pair of Create Particles Commands to Create both Emitter and Sheath Electrons

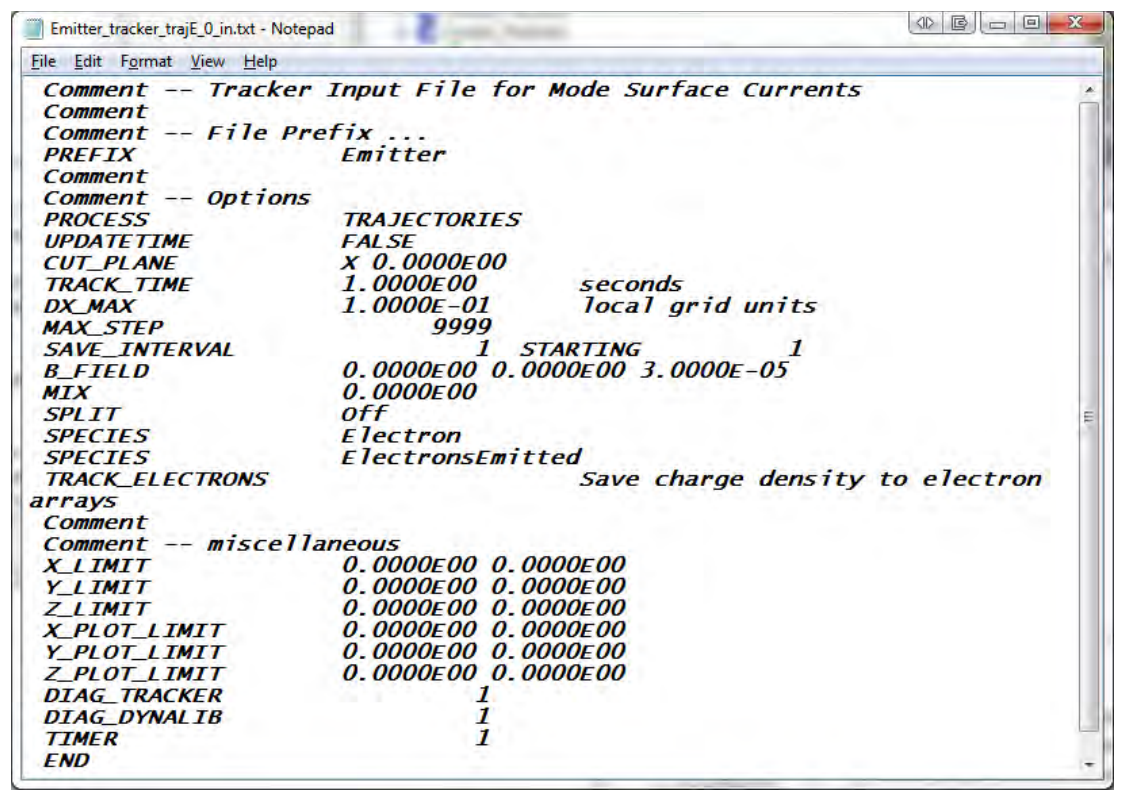

Figure 24. Edit of Tracker Input file. Note That Both Species (Sheath Electrons and Emitter Electrons) are Specified for Tracking

\subsubsection{Results}

The resulting potential versus time is shown in Figure 25. Early on, the charging rate is limited by the $\mathrm{dJ} / \mathrm{dV}$, which is set to $0.75 \mathrm{~J} / \mathrm{V}$. The potential increases until the sheath current balances the emitter current at about $840 \mathrm{~V}$. The charging rate continues to be limited because the code sets 
$\frac{d I}{d V}=\sum_{\text {elem }} \frac{d J}{d V}=-0.75 \sum_{\text {elem }}\left|\frac{J}{V}\right|<<-0.75\left|\frac{I}{V}\right|$. i.e, $\mathrm{dI} / \mathrm{dV}$ is large and negative even though I is near zero due to cancellation of the emitted and sheath electrons.

The net current versus potential is shown in Figure 26. At about $840 \mathrm{~V}$ the sheath electron current cancels the emitter current. Note that the net current is obtained by taking the average "Tracked Electron Current" to all surface elements and multiplying by the total area of 1.4823 $\mathrm{m}^{2}$.

If the option is used to set the stabilizing current derivatives to zero, then the correct (using the vacuum capacitance) charging timescale is achieved, as shown in Figure 27.

Potential

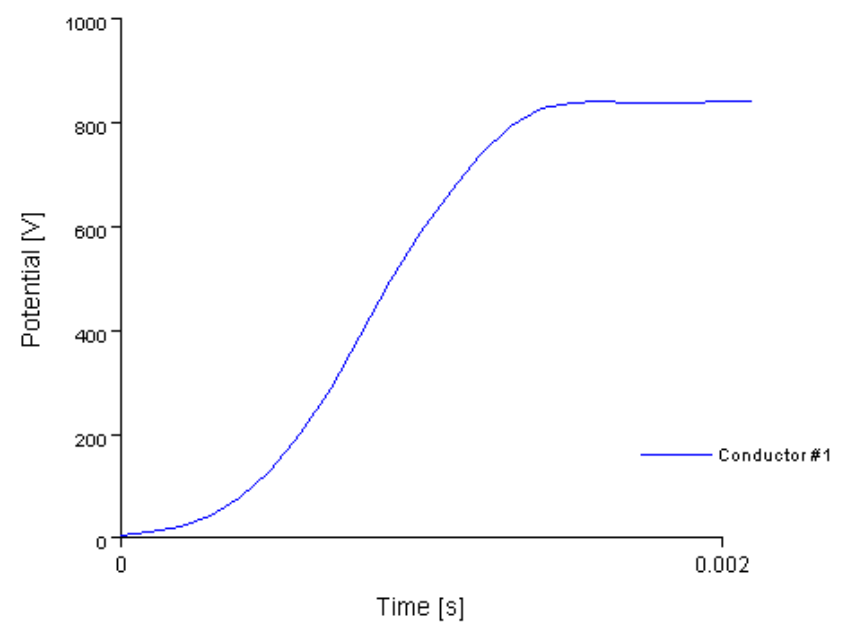

Figure 25. Potential versus Time for the Emitter Problem

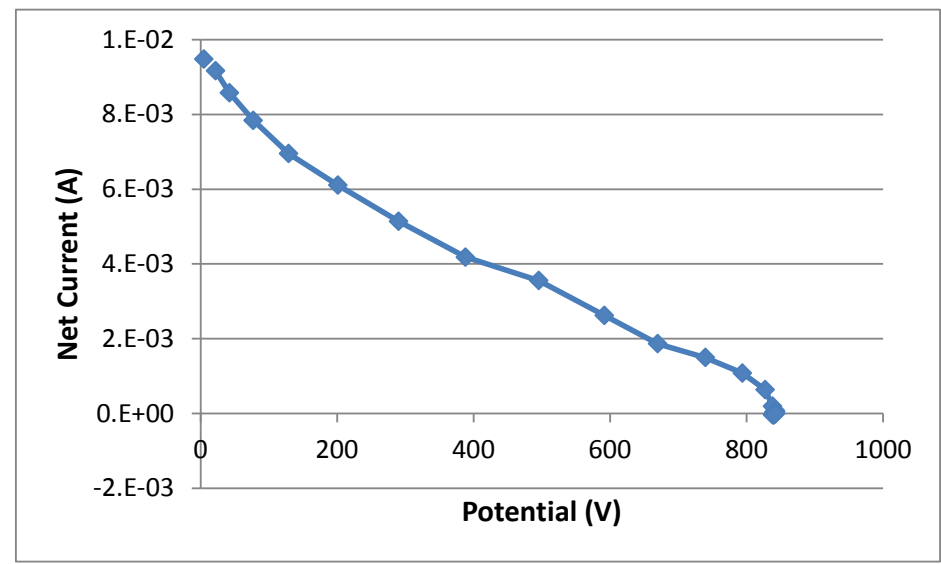

Figure 26. Net Current versus Potential. 


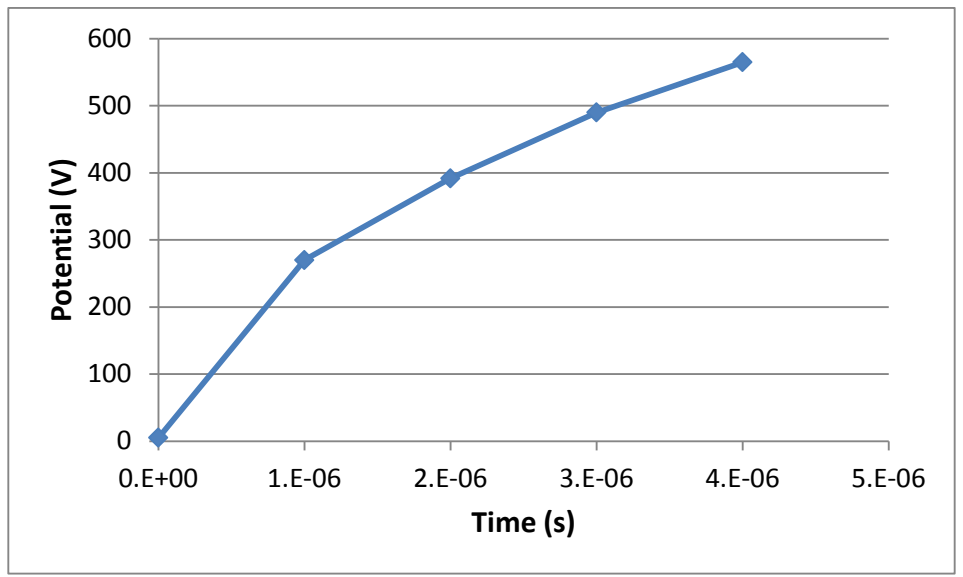

Figure 27. Charging Dynamics with the Stabilizing Current Derivatives Set to Zero

\subsection{Detector Test Case}

In order to explore and test Nascap-2k's ability to model a detector, the current to a detector with a guard ring was computed.

This test case has been added to the Nascap-2k User's Manual as an example.

\subsubsection{Object}

The object, intended to represent a detector with a rudimentary collimator, is shown in Figure 28. The detector (cyan surface element) and the surrounding eight surface elements (guard ring) are defined as conductor 3 , and are biased $+5 \mathrm{~V}$ relative to the remainder of the plate and the collimator (yellow surface elements). The definition of the detector is shown in Figure 29. It specifies the emission of test particles from four locations on the detector in 144 directions and with 20 energies ranging from $5 \mathrm{eV}(0 \mathrm{eV}$ of total energy) to $7 \mathrm{eV}$ ( $2 \mathrm{eV}$ of total energy).

\subsubsection{Problem Tab}

On the Problem tab, the environment is specified as "LEO or Plume" and the problem type as

"Potentials in Space or Detector Analysis" with "Analytic Space Charge" and "Surface Currents". 


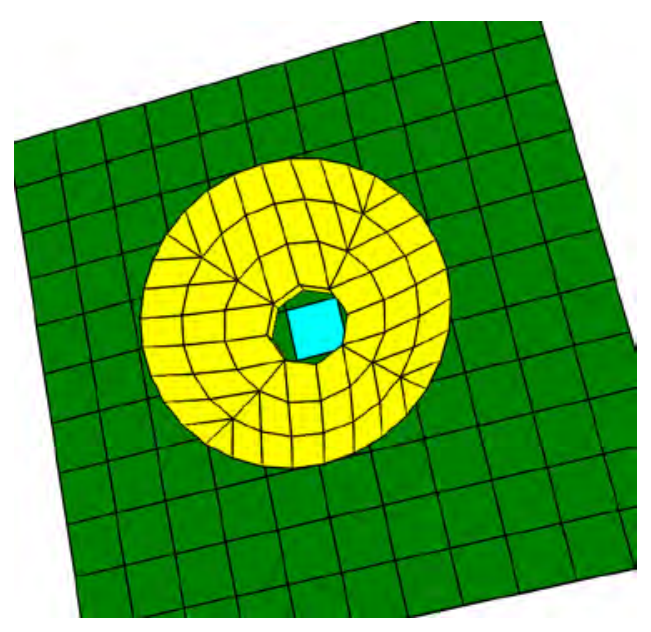

Figure 28. Detector Test Case Object.

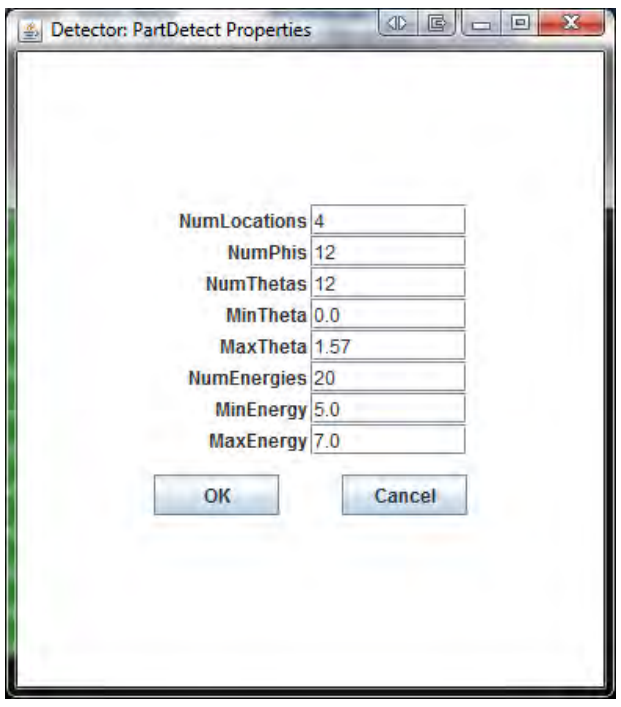

Figure 29. Definition of the Detector Properties

\subsubsection{Environment Tab}

The Environment tab specifies a plasma with density $10^{11} \mathrm{~m}^{-3}$, temperature $0.3 \mathrm{eV}$, and no motion or magnetic field.

\subsubsection{Applied Potentials Tab}

On the Applied Potentials tab, conductor 1 is fixed at $0 \mathrm{~V}$ (ground), and conductor 2 (collimator) and conductor 3 (detector and guard ring) are biased at 0 and $+5 \mathrm{~V}$ respectively.

\subsubsection{Space Potentials Tab}

On the Space Potentials tab, the "Non-linear" potential formulation is chosen. 


\subsubsection{Particles Tab}

The Particles tab (Figure 30) specifies electron emission from a surface using the "Detector" treatment, with specifications for the "PartDetect" detector that was defined during object definition by Object Toolkit.

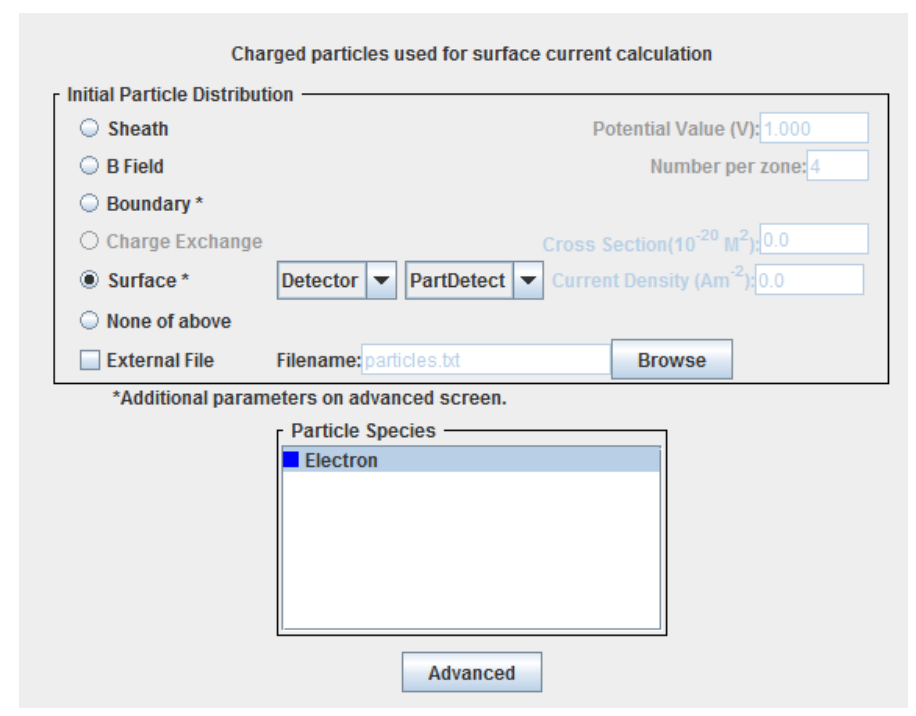

Figure 30. Particles Tab for the Detector Simulation

\subsubsection{Script Tab}

With the above settings, the default script, Figure 31, is used. Run the script to obtain the results discussed in the following sections.

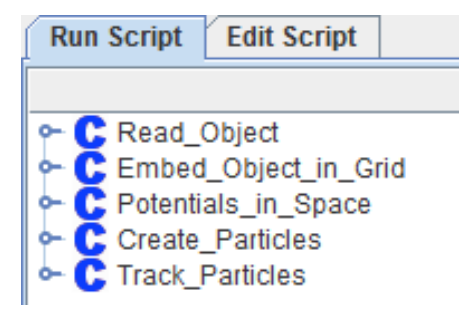

Figure 31. Script for the Detector Problem (Default Script)

\subsubsection{Results Tab}

With no charging and only one surface element with current, there is very little information to be viewed on the Results tab, seen in Figure 32. It is possible to see that one surface element (the detector surface element) is assigned a current density of $-3.257 \mathrm{~mA} \mathrm{~m}^{-2}$. 


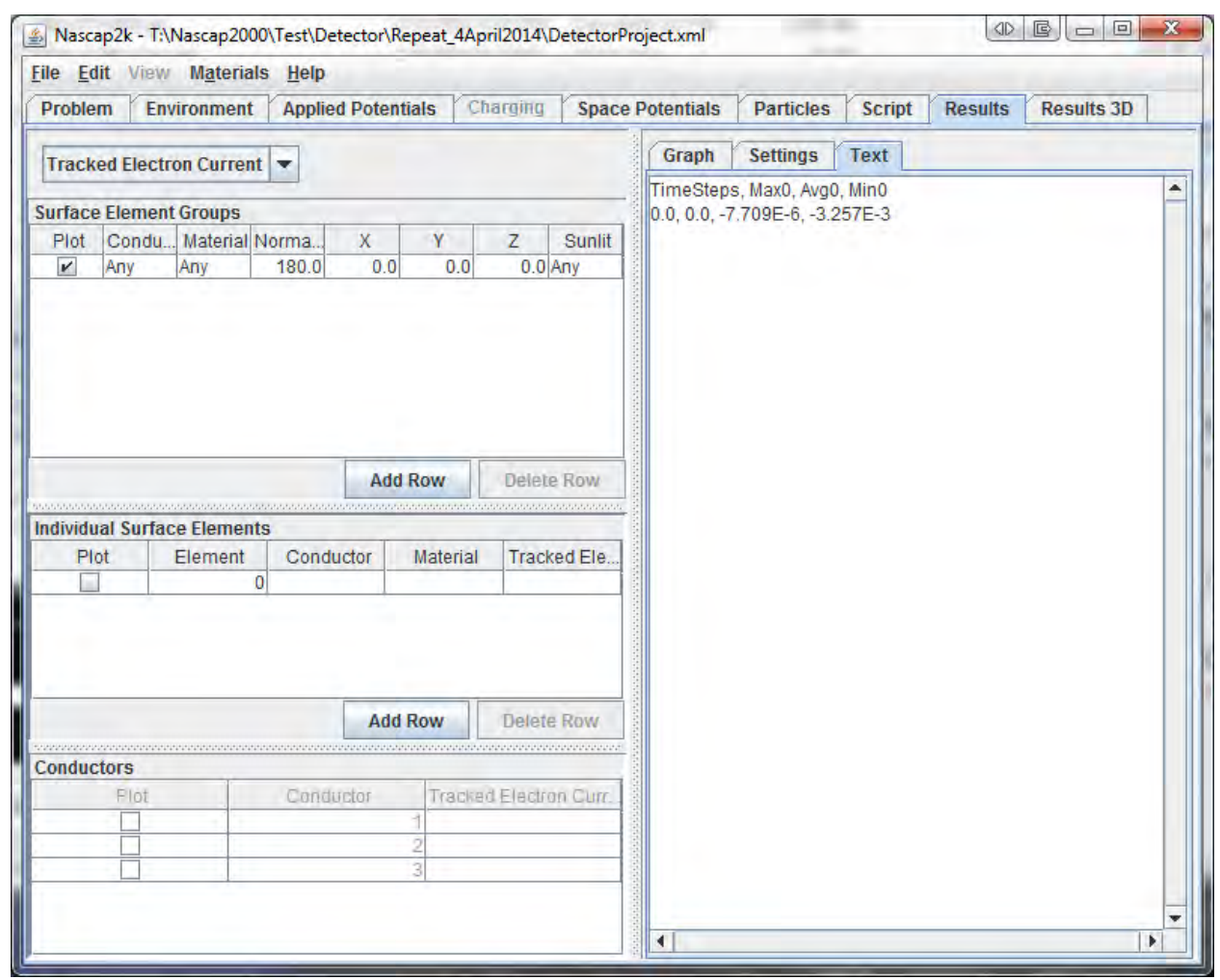

Figure 32. Results Tab Following Execution of the Detector Problem

\subsubsection{Results 3D Tab}

The Results 3D tab, shown in Figure 33 is used to obtain the results. The properties of the detector surface element confirm the current density noted above. Multiplying by the surface element area gives $4.0 \mu \mathrm{A}$ as the current to the detector. The potential bows out significantly from the collimator aperture, suggesting that the current is somewhat greater than the aperture area times the electron thermal current. Since the aperture approximates a circle of $2.5 \mathrm{~cm}$ radius and the electron thermal current is $1.468 \mathrm{~mA} \mathrm{~m}^{-2}$, the planar current through the aperture would be only $2.9 \mu \mathrm{A}$. (By comparison, a sheath calculation with the sheath potential set to $0.2 \mathrm{~V}$ gives $3.4 \mu \mathrm{A}$ to the detector surface element.)

\subsubsection{Tracker Output}

The Tracker output, the relevant excerpt of which is shown in Figure 34, sheds additional light on the calculation. Of 11520 test particles launched from the detector surface element, 1365 left the primary grid (meaning that they represent electrons from the environment), while 10155 struck object surfaces (meaning they represent phase space that does not connect to the environment). Applying environmental factors to the "lost" current particle weights yields the 4.3 $\mu \mathrm{A}$ noted above, and is assigned to conductor 3 "alum" surface elements (representing the detector). Applying environmental factors to the 10155 test particles that struck the object yields $5.14 \mu \mathrm{A}$; this is not necessarily a meaningful number as these particles do not represent electrons from the environment, but gives a better estimate than the raw particle numbers or weights of how much of the detector's phase space is blocked. 

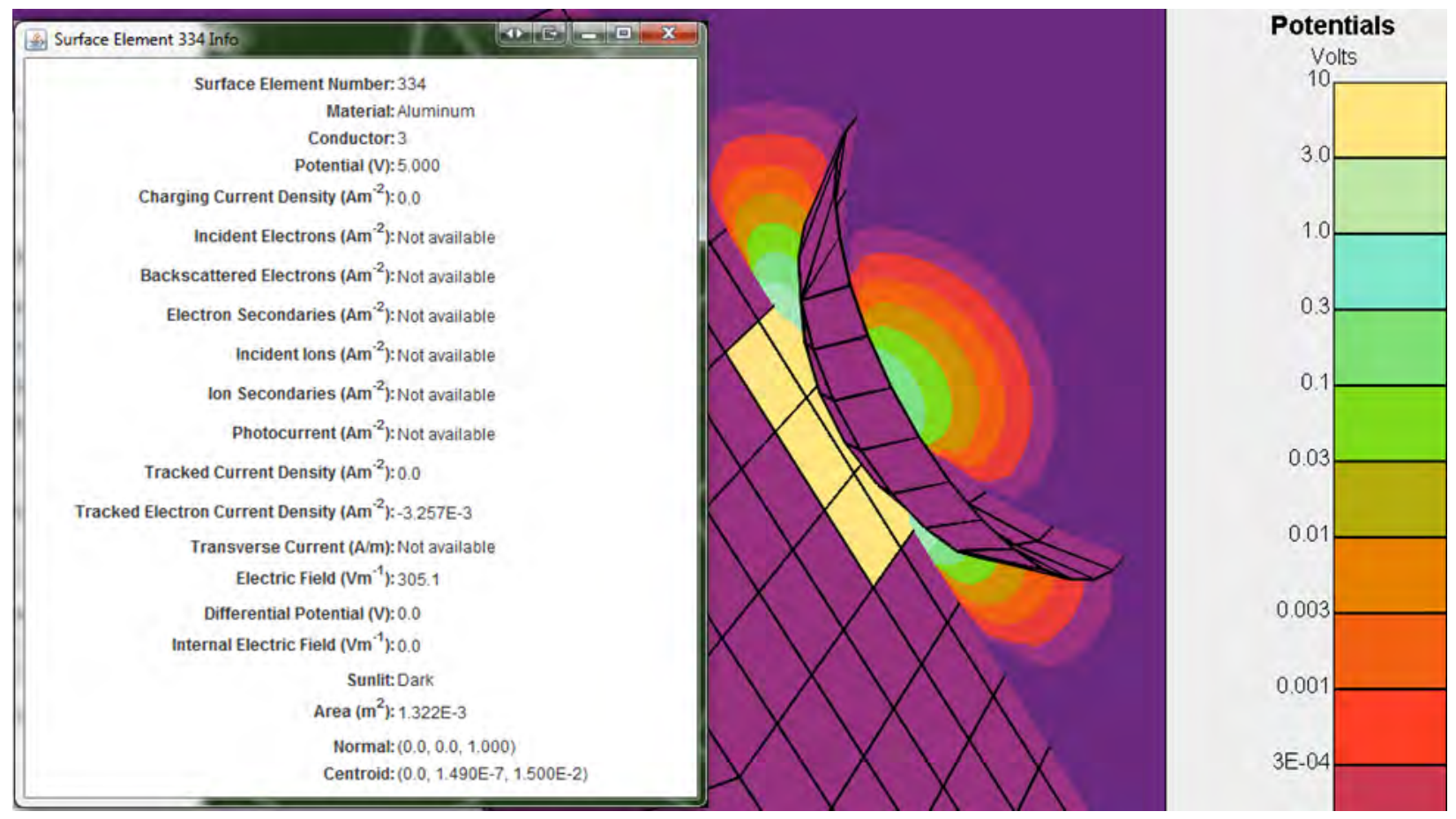

Figure 33. Results 3D Tab, Showing Properties of Detector Surface Element, and Showing Bowing Out of Potentials through the Collimator

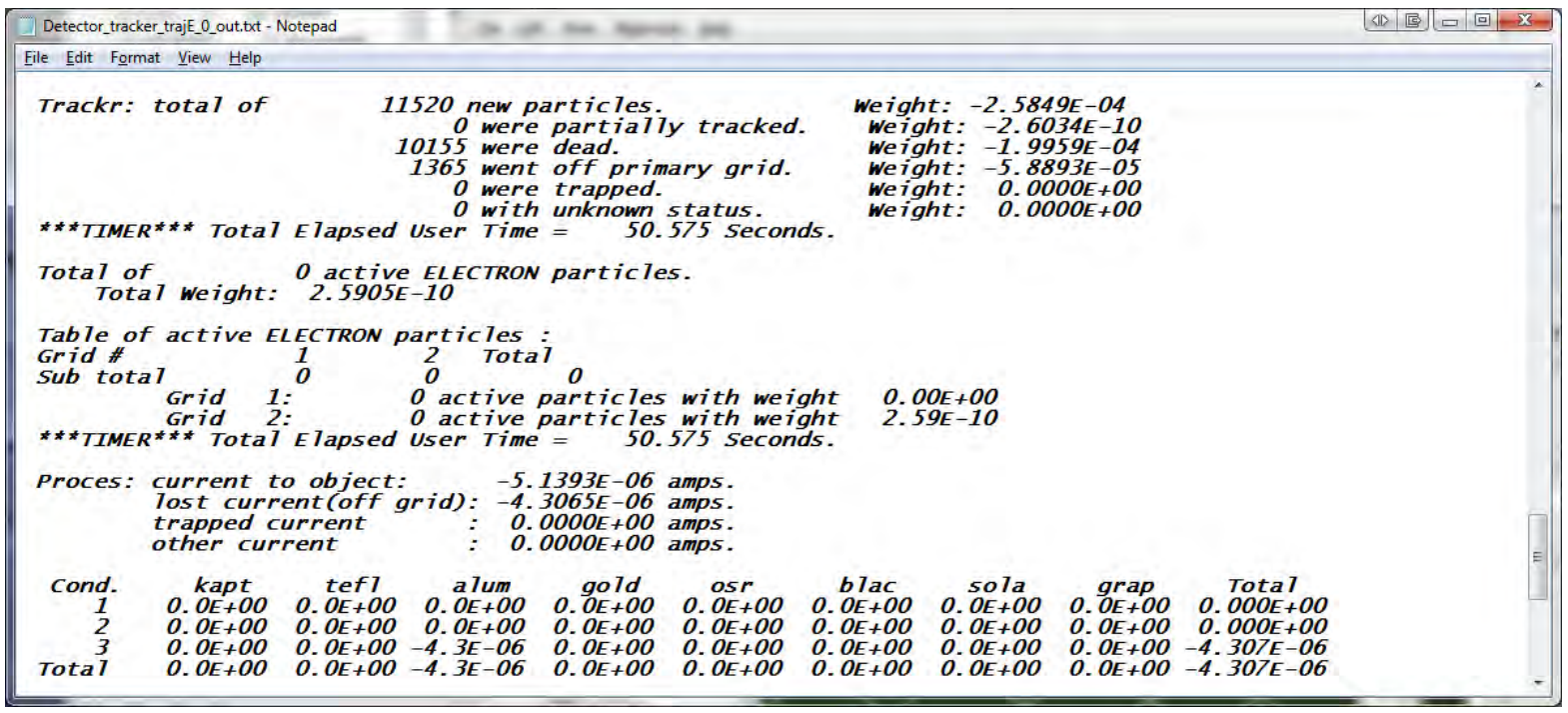

Figure 34. Tracker Output from Detector Run

Approved for public release; distribution is unlimited. 


\section{MEMO SUMMARIZING RESOURCES FOR INTERNAL CHARGING COMPUTATIONS}

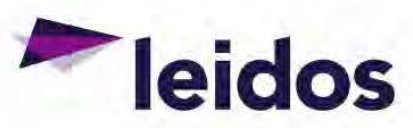

Date: January 23, 2014

To: Dale Ferguson and Adrian Wheelock

From: Victoria Davis and Myron Mandell

Subject: Summary of Resources Regarding Internal Charging Environment and Internal Charging Computation

The following is a compilation of resources we have used at times in assessing risk due to Internal Charging.

\section{Proposed Worst Case and Design Environments}

\section{Fennell Environment}

This environment is described in a paper by Joe Fennell [Fennell et al., 2000]. The paper proposes a worst case spectrum for geosynchronous orbit based on measurements taken after the March 24, 1991 magnetic storm event. They also propose a HEO/Molniya worst case spectrum.

\section{Flumic}

Flumic was developed at QinetiQ and is documented in two papers, [Wrenn, et al., 2000] and [Rodgers et al., 2003]. The model has been validated by a comparison with flight data [Evans, et al, 2007] and [Taylor, et al, 2009]. Flumic is included in Spenvis for use with DICTAT. Victoria has the formulas implemented in an Excel spreadsheet. The Flumic spectrum depends on L, solar cycle, and season. The inner belt model also has a $\mathrm{B} / \mathrm{B}_{0}$ dependence. In general, it gives more internal charging than other models due to the spectral shape.

\section{AE8}

AE8 is a climatological average of the environment. As such, it is useful for estimating degradation of solar cells over a period of years. It is sometimes used to estimate internal charging after enhancement by a factor on the order of ten.

\section{NASA-HDBK-4002}

The Handbook has a figure showing a proposed worst case environment for internal charging on geosynchronous spacecraft. At one time, Victoria read the numbers off the figure and has done calculations using it.

\section{Frederickson fit}

Robb Frederickson had the differential fluence over the CRRES five-hour half-orbits fit to a functional form. These fits are tabulated in a NASA report [Frederickson and Brautigam, 2004]. Victoria determined that the orbit 600 spectrum gives the most internal charging. 


\section{Software to Compute Deep Dielectric Charging}

\section{NUMIT}

Several versions of Robb Frederickson's Fortran code NUMIT exist. Joe Minow, Insoo Jun, and Brian Beecken have all evaluated the code and made modifications. I believe that David Cooke has Brian Beecken on contract to create a standard version. Insoo Jun announced a similar effort recently.

\section{SAIC Deep Dielectric Charging Code}

The Leidos proprietary code is a more flexible stand-alone version of the code originally implemented in the SEE Spacecraft Charging Handbook. It implements the same algorithms as NUMIT and Robb was heavily involved in the original implementation. It has been verified to give the same results as NUMIT. It is written in Java. See [Davis, et al., 2007].

\section{DICTAT}

The Spenvis tool was developed at QinetiQ (then DERA). See on-line documentation and [Rodgers, et al., 1998].

\section{Anomaly Investigations}

\section{Violet and Frederickson}

An anomaly investigation that doesn't get the attention it deserves was done by Mike Violet for Robb Frederickson [Violet and Frederickson, 1993].

\section{SEAES-GEO}

This expert system [O'Brien, 2009] uses the 12 hour average of the electron flux over $2 \mathrm{MeV}$ measured by GOES to predict the anomaly risk. The formula is based on the correlation of electrostatic discharges measured by SCATHA with the 12 hour average of the over $1810 \mathrm{keV}$ energy channel of the SC3 instrument. The tool does include a mapping from the GOES location to the spacecraft location.

\section{References}

V.A. Davis, M.J. Mandell, R.H. Maurer, Preliminary Surface and Internal Charging Analysis of the Radiation Belt Storm Probes Spacecraft, $9^{\text {th }}$ Spacecraft Charging Technology Conference, Biarritz, France, 2007.

H.D.R. Evans, D.J. Rodgers, E.J. Daley, P. Nieminen, A. Mohammadzadeh, P. Buehler, W. Hajdas, Energetic Electron Results from the ESA Monitors and Comparison with Existing Radiation Belt Internal Charging Models, $9^{\text {th }}$ Spacecraft Charging Technology Conference, Biarritz, France, 2007.

J.F. Fennell, H.C. Koons, M.W. Chen, J.B. Blake, Internal Charging: A Preliminary Environmental Specification for Satellites, IEEE Trans Plasma Science, 28, p. 2029, 2000.

A.R. Frederickson and D.H. Brautigam, Mining CRRES IDM Pulse Data and CRRES Environmental Data to Improve Spacecraft Charging/Discharging Models and Guidelines, NASA/CR-2004-213228, 2004. 
T.P. O'Brien, SEAES-GEO: A Spacecraft Environmental Anomalies Expert System for Geosynchronous Orbit, Space Weather, 7, doi:10.1029/2009SW000473, 2009.

D.J. Rodgers, K.A.Ryden, G.L.Wrenn, P.M.Latham, J. Soringrensen, L.Levy, An Engineering Tool for the Prediction of Internal Dielectric Charging, $6^{\text {th }}$ Spacecraft Charging Technology Conference, Hanscom, MA, 1998.

D.J. Rodgers, K.A. Hunter, G.L. Wrenn, The FLUMIC Electron Environment Model, $8^{\text {th }}$ Spacecrafi Charging Teehnology Conference, Huntsville, AL, 2003.

B. Taylor, C.I. Underwood, K.A. Ryden, P.A. Morris, A GIOVE Derived Galileo Electron Spectrum and Comparison to Models, IEEE Trans Nuclear Science, 56, p. 3423, 2009,

M.D. Violet and A.R. Frederickson, Spacecraft Anomalies on the CRRES Satellite Correlated with the Environment and Insulator Samples, IEEE Trans Nuclear Science, 40, p. 1512, 1993.

G.L. Wrenn, D.J. Rodgers, Modeling the Outer Belt Enhancements of Penetrating Electrons, J. Spacecrafi and Rockels, 37, p, 408, 2000. 


\section{MEMO SUMMARIZING RESOURCES FOR AURORAL CHARGING COMPUTATIONS}

\section{leidos}

Date: February 4, 2014

To: Dale Ferguson and Adrian Wheelock

From: Victoria Davis and Myron Mandell

Subject: Summary of Resources Regarding Auroral Charging Environment Characterization and Charging Computation

From a surface charging perspective, the auroral environment is poorly characterized. There exists quality data that could be used to fill this gap.

\section{DMSP}

The best correlation study between environment measures and spacecraft potentials we are aware of is that done by Paul Anderson ${ }^{1}$. From an examination of 12 years of DMSP data, he concluded that the DMSP spacecraft charged more than $100 \mathrm{~V}$ negative under the following conditions.

- The spacecraft was in darkness;

- The plasma density was less than $10^{4} \mathrm{~cm}^{-3}$; and

- There was a high integral number flux $\left(>10^{8}\right.$ electrons $\left.\mathrm{cm}^{-2} \mathrm{~s}^{-1} \mathrm{~s}^{\mathrm{r}-1}\right)$ of high energy $(>14$ $\mathrm{keV})$ electrons.

Mengu Cho also did a statistical study of DMSP environment data. ${ }^{2} \mathrm{He}$ used data from the environment sensors for three selected years, filtered it for availability of full spectra and a quality measure, and fit the spectra to a double Maxwellian. He then categorized the fits by resulting densities and currents and determined the frequency of the electron current from the fit exceeding the ion current from the fit as a function of year, season, flux of high energy electrons (based on fit), and pole. With additional assumptions, he then used the results in MUSCAT to construct a frequency of charging estimate. He appears to have not included any information regarding the measured charging.

David Cooke has proposed a worst case environment for charging calculations. It is based on a fit to a measured environment during an auroral charging event. If I remember correctly, the low energy plasma density was then reduced from the measured value.

\section{Freja}

The Freja team recorded 39 charging events at high altitude over the northern aurora. The spacecraft passed over the southern aurora at much lower altitude, but no data was recorded. ${ }^{3}$

- No charging events were observed for cold plasma density above $2 \times 10^{3} \mathrm{~cm}^{-3}$.

- The record potential was nearly $-3 \mathrm{kV}$, during which the measured ion density was about $30 \mathrm{~cm}^{-3}$.

- Most events occurred when the spacecraft was sunlit. However, the ionosphere below was in darkness. 
POLAR calculations using a representation of the Freja geometry and materials and fits to measured environments were done. ${ }^{4}$ The calculations predicted much less charging than observed. One known issue with these calculations is that the power law component of the Fontheim was extended down to $0.5 \mathrm{~V}$, well below the energy range of the measured spectrum. Thus the calculations had large numbers of low energy electrons that were (presumably) not actually present.

\section{Possible Charging Index Studies}

The Freja calculations could be redone using improved fits and Nascap- $2 k$.

A comparison of measured chassis potential and calculated chassis potential of DMSP for a selection of events could be done, with the goal of arriving at a proposed worst case environment.

A study similar to that we did with the LANL data with the goal of determining the best spectral shape to use for charging studies would be very interesting. Such a study will be most efficient if it involved Paul Anderson. The last time such a study was contemplated, it was determined that it would take significantly more than the funds presently allotted to us for the charging index study.

\section{References}

${ }^{1}$ P.C. Anderson, Characteristics of Spacecraft Charging in Low Earth Orbit, J Geophys Res, 117, doi: 10.1029/2011JA016875, 2012.

${ }^{2}$ M. Cho, K. Saito, T. Hamanaga, Data Analysis of the Polar Plasma Environment for Spacecraft Charging Analysis, Acta Astronautica, 81, p. 160, 2012.

${ }^{3}$ A.I. Eriksson and J.-E. Wahlund, Charging of the Freja Satellite in the Auroral Zone, IEEE Trans Plasma Science, 34, p. 2038, 2006.

${ }^{4}$ A.I. Eriksson, L. Wedin, J.-E. Wahlund, B. Holback. Analysis of Freja Charging Events: Modeling of Freja Observations by Spacecraft Charging Codes, SPEE-SP120-TN-,1999. 


\title{
7. SEMI-ANALYTIC AND PIC (PARTICLE-IN-CELL) METHODS FOR QUANTIFYING CHARGING IN DENSE, COLD PLASMA
}

The following paper was prepared for the $13^{\text {th }}$ Spacecraft Charging Technology Conference in Pasadena, CA in June 2014.

\section{Semi-analytic and PIC (Particle-in-cell) Methods for Quantifying Charging in Dense, Cold Plasma}

\author{
V.A. Davis, M.J. Mandell, D.L. Cooke, D.C. Ferguson
}

\begin{abstract}
Researchers use several techniques for the calculation of sheath structure and surface currents in short Debye length plasma for the purpose of calculating surface potentials of complex spacecraft. These techniques include the assignment of analytic results for simple geometries, semianalytic methods that use Poisson's solution with an analytic charge density formula in conjunction with particle tracking, hybrid-PIC (particle in cell) techniques in which the charge density is determined by tracking ions and assuming Boltzmann electron densities, and full PIC. Hybrid-PIC and full PIC techniques can be applied to both static and dynamic plasmas.
\end{abstract}

We compare the various techniques available in the plasma modeling code Nascap- $2 k$, along with the analytic approach used in the analysis tool EWB. We review the strengths, weaknesses. and limitations of the available models. In the appropriate limits, each approach gives the analytic result-within the accuracy of the calculation. When used under conditions outside the limits of the approximations, results are not reliable and may be misleading. At the same time, the PIC and hybrid PIC approaches can be misleading when phase space is not adequately sampled.

When used within their range of applicability. these techniques are powerful tools in assessing charging in short Debye length plasma.

Keyworils-charging; modeling; dense plasma

\section{Charging MFCHANISMS}

This paper surveys the various approaches used to model spacecraft charging in cold, dense plasma, such as found in low-Earth-orbit. The range of plasma properties under consideration are listed in Table I. In these plasmas, with a few exceptions (such as when thin dielectrics lead to high surface capacitances), the current is high enough that spacecraft surface potentials adjust to changes in the spacecraft and environment within milliseconds. Dynamic effects are associated with wake effects, result from switching of solar arrays on and off, and occur in dynamic experiments. However, the main interest, and the focus of this paper, is the calculation of steady-state potentials.

V.A. Davis and M.J. Mandell are with Leidos, Ine., San Diego, Califomia. USA fe-mail, victoria a.davis a leidos,com, myron,j. mandell â leidos.com

D.1. Cooke and D.C. Ferguson are with the Air Force Researeh

Laboratory/RVB, Albuquerque. New Mexico, USA

(david cookeâkinland.af.mil, dale.fergusona kintland af.mil)

This research was earried out under coniract with the Air Force Research Labonatory, FA9453-11-C-0262

T2014 Leidos. All rights rescrved

\begin{tabular}{|c|c|}
\hline Femperature & $0.1105 \mathrm{cV}$ \\
\hline Density & $10^{8}$ to $10^{12} \mathrm{~m}^{\prime}$ \\
\hline Debye length & 0.2 to $50 \mathrm{~cm}$ \\
\hline Electron thermal current & $9 \mu \mathrm{A} \mathrm{m}^{2}$ 10 $60 \mathrm{~mA} \mathrm{~m}$ \\
\hline Oxygen thermal curren & $0.05 \mu \mathrm{Am}$ to $0.3 \mathrm{~mA} \mathrm{~m}^{2}$ \\
\hline Hydrogen thermal current & $0.2 \mu A m^{2}$ to $1 \mathrm{~mA} \mathrm{~m}^{4}$ \\
\hline Ram currem & $1 \mu \wedge m^{2}$ is $1 \mathrm{~mA} \mathrm{~m}^{2}$ \\
\hline
\end{tabular}

In low energy plasma, the net surface current is dominated by the incident charged particles. This is different from higher energy, more tenuous plasmas, such as geosynchronous substorm and auroral environments. In cold plasma the energy of the incident charged particles is low, so few secondary electrons are created, and (except in the more tenuous environments) even the ion thermal current exceeds the photoemitted electron current.

In low-Earth orbit the spacecraft velocity is on the order of $7 \mathrm{~km} / \mathrm{sec}$, slow compared with the electron thermal speed, but fast compared with the thermal speed of an atomic oxygen ion. The ion and electron densities immediately behind the spacecrafi are dramatically reduced because only ions with velocities comparable to the spacecraft velocity can exist in this "deep wake" region. The electron density would be near uniform were it not for the negative potential due to electron space charge from the excess electrons. In this mesothermal plasma, the ion current, and to a much lesser extent the electron current, depends on the angle of the surface with respect to the motion.

Significant charging in cold plasma is driven by spacecraftgenerated voltages. The resulting potentials are of the same order. A common example of spacecraft-generated voltages is the positively biased interconnects and bus bars of the solar arrays that are exposed on most spacecraft. These electrodes collect electrons from the surrounding plasma, driving the spacecraft ground negative, generally to about $90 \%$ of the entire solar-array-generated voltage. The floating potential is determined by the ion current to other exposed conductive surfaces. The electron current collected by all the positive potential conducting surfaces (such as the interconnects) is balanced by the ion current collected by the negative potential conducting surfaces.

In the absence of spacecraft-generated potentials and on insulating surfaces, the surface potential is determined by the I

Approved for public release; distribution is unlimited. 
balance between the attracted species and the repelled species. Thus surfaces float negative a few times the plasma temperature, so that the attracted ion current is balanced by the repelled electron current. Under unusual conditions, potentials on the order of the ion kinetic energy can develop in order to repel the ion flux.

\section{AVAII.ABLE TECHNIQUES}

The closely related techniques of current balance and charging are used to compute the equilibrium potential of surfaces in cold, dense plasma. In current balance, the net current as a function of surface potentials is computed in order to find the zero of the current-voltage relation. When the charging technique is used, initial surface potentials are assumed. The resulting surface currents and an assumed capacitance are used to determine the change in surface potentials. The process is then iterated until the zero current condition is reached. In both approaches, the current as a function of surface potential is the central parameter.

The current computations depend on how the plasma is modeled. There are four basic approaches, along with several variations.

- Analytic currents

a Currents computed using a formula

- Particle Tracking/Analytic space charge

a Poisson's equation solved with space charge specified by an analytic formula

- Current of one or both species computed by tracking macroparticles.

- Hybrid PIC

- Poisson's equation solved with space charge given by ion densities from macroparticle tracking and electron densities from an analytic formula.

o Current of one or both species computed by tracking macroparticles

- PIC

Each of these approaches has benefits and drawbacks. While analytic approaches are faster and more stable, the underlying approximations may or may not be applicable. Selfconsistent PIC calculations include all the physical phenomena, but require care to adequately represent the phase space distribution function and are computationally intensive.

\section{A. Analytic currents}

In this approach, formulas for plasma currents are derived by assuming an idealized geometry and plasma and calculating an exact answer. A major advantage is that once a formula has been developed, applying it to a new system is quick and easy. The applicability of a formula to other conditions is limited by how close the system under consideration is to the ideal. Generally these formulas depend on local values such as the surface potential and orientation with respect to the direction of motion, and sometimes the surface electric field. Here we discuss two common and one not-so-common approximations. In the plasmas of interest, in the absence of spacecraft driven changing surface potentials, it is reasonable to assume that the plasma is at equilibrium and therefore, is Maxwellian.

\section{1) Planar}

In the absence of motion, if a negative potential collecting surface is very large compared to the Debye length, the attracted current is the ion thermal current and the repelled current is the electron thermal current reduced by the Boltzmann factor. This condition can be used to determine the floating potential of an isolated object.

$$
n e \sqrt{\frac{e T}{2 \pi m_{1}}}=n e \sqrt{\frac{e T}{2 \pi m_{e}}} \exp \left(\frac{\phi}{T}\right)
$$

For an oxygen plasma, the solution to this equation is $\phi=-5.14 T$, five times the temperature.

When the collecting surface is in motion, the thermal current is multiplied by a velocity dependent angular factor on the ram side. The simplest approximation is to use the ion ram current times a cosine of the angle with respect to flow for the ion current and the same factor as above for the electron current.

$$
n e v_{i} \cos \theta_{\text {ram }}=n e \sqrt{\frac{e T}{2 \pi m_{e}}} \exp \left(\frac{\phi}{T}\right)
$$

For a surface directly facing the ram at a typical low-Earthorbit speed of $7.500 \mathrm{~m} / \mathrm{s}$ in a $0.1 \mathrm{eV}$ plasma, the surface is at $-0.195 \mathrm{~V}$, twice the temperature.

\section{2) Flowing Maxwellian}

We can calculate the current to a surface in motion in the orbit-limited approximation (flowing Maxwellian). The orbitlimited approximation is commonly used when modeling tenuous plasmas. It is strictly correct for a $1 / \mathrm{r}$ potential field with no physical or electrostatic barriers.[1] It is appropriate for cases in which the Debye length is significantly larger than the spacecraft size, and therefore, while it is often useful as a first estimate, the results must be confirmed by other techniques when used in dense, cold plasma. The current is given by the following expression [2]

$j=q \int_{1}^{\infty}\left(\frac{E}{E \pm \phi}\right) \sqrt{\frac{e}{2 \pi}} \frac{E}{T} n \operatorname{nexp}\left(-\frac{E+m U^{2} / 2-\sqrt{E m / 2} U / \cos \psi}{T}\right) d E$

where the integration variable $E$ represents the energy at the surface, the integral limit $L$ is 0 for the repelled species and $|\phi|$ for the attracted species, the fraction in parentheses represents the orbit limited enhancement or reduction of the current, the upper sign is for ions and the lower sign is for electrons, $U$ is the velocity of the plasma, and $\psi$ is the angle between the flow vector and the incident velocity at infinity, which can be related to the velocity at which the particle strikes the surface. In the absence of motion, this formula reduces to a Maxwellian.

In the orbit-limited approximation, the potential of a $1 \mathrm{~cm}$ radius conductive stationary sphere in a $10^{11} \mathrm{~m}^{-3}, 0.1 \mathrm{eV}$ oxygen plasma $\left(\lambda_{\mathrm{D}}=0.7 \mathrm{~cm}\right)$ is $-0.36 \mathrm{~V}$, less negative than in the planar approximation. The floating potential of the same sphere moving at a typical low Earth orbit velocity of $7.5 \mathrm{~km} / \mathrm{sec}$ is $-0.324 \mathrm{~V}$, slightly less, as the higher ion current 
in the ram more than makes up for the lower ion current in the wake.

\section{3) Sheath}

In dense plasma with high potentials, the disturbed region is referred to as a sheath. In the sharp sheath edge approximation, the sheath edge represents a demarcation between a low potential exterior region containing neutral undisturbed plasma and a high potential interior region from which one species is excluded. The charge of the attracted species in the sheath region balances the charge on the spacecraft surfaces.

Consider a simple system of two conductive spheres biased with respect to each other. The spheres are $10 \mathrm{~cm}$ in radius and their centers are $40 \mathrm{~cm}$ apart. Fig. 1 shows the potentials in a plane through their centers with the sphere potentials set to $+50 \mathrm{~V}$ and $-50 \mathrm{~V}$. In this calculation, the plasma is $10^{11} \mathrm{~m}^{-3}$, $0.2 \mathrm{eV}$ giving a Debye length of $1.05 \mathrm{~cm}$, short compared with the sphere size and separation. The ions are atomic oxygen and the spheres are stationary. Within each sheath, the repelled species is excluded leaving behind the charge of the attracted species to balance the charge on the sphere. The overlapping sheaths extend about $10 \mathrm{~cm}$ out from the spheres. Outside of that region the potentials are low.

When a sheath forms, the current to an object can be estimated as the current to its sheath. This is the approach used by EWB [3] (Environment WorkBench). In this approximation, each component is surrounded by a sheath of radius $r_{s c}$ where $r_{s c}$ is the solution to the equation

$$
\frac{\phi}{T}=0.8356\left(\frac{r_{s c}}{\lambda_{D}}\right)^{4 / 3}\left(\left(\frac{r_{s c}}{a}\right)^{3 / 4}-\left(\frac{a}{r_{s c}}\right) 0.17\right)^{4 / 3},
$$

where $a$ is the radius of a sphere with the same surface area as the collecting area of the component. This formula is based on earlier work by Parker.[4] The sheath surfaces are then divided

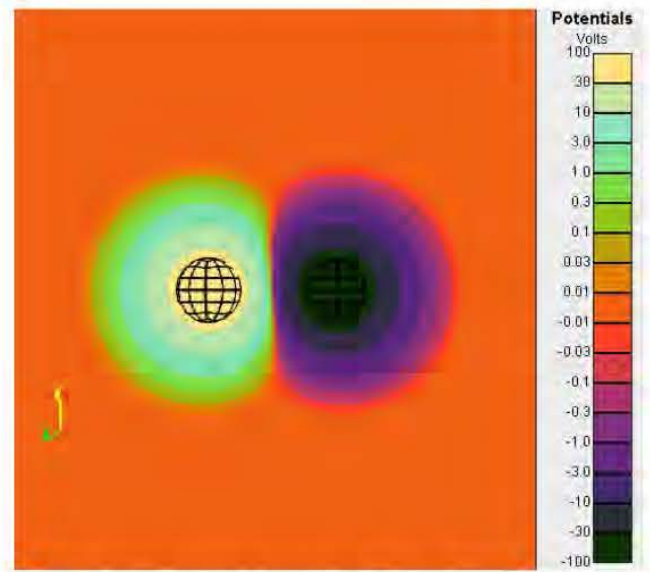

Fig. 1. Illustration of potentials on plane through center of stationary two-sphere object with spheres are $+50 \mathrm{~V}$ and $-50 \mathrm{~V}$, in $10^{11} \mathrm{~m}^{-2}, 0.2$ $\mathrm{eV}$ oxygen plasma. into several parts. Portions of the sheath that overlap are discarded. The current to each sheath segment is determined by the low potential formula above. This approach works well when the problem is sheath dominated. In this model, the floating potentials of the two spheres are $-19.6 \mathrm{~V}$ and $+80.4 \mathrm{~V}$.

\section{B. Tracked currents in potentials computed with analytic space charge}

A more computationally intensive approach is to numerically solve Poisson's equation using an analytic formula for the space charge and then track charged particles in the resulting space potentials. One such approximation that is applicable at both high and low potentials is the following non-linear formula[5]

$$
\begin{gathered}
\frac{\rho}{\varepsilon_{0}}=-\frac{\phi}{\lambda_{D}} \frac{\max (1, C(\phi, E))}{1+\sqrt{4 \pi}|\phi / T|^{3 / 2}} \\
C(\phi, E)=\min \left((R / r)^{2}, 3.545|\phi / T|^{3 / 2}\right) \\
(R / r)^{2}=2.29\left|E \lambda_{D} / T\right|^{1.262}|T / \phi|^{0.509}
\end{gathered}
$$

where the symbols refer to the local potential, $\phi$, and the local electric field, $E$. This function smoothly interpolates between linear Debye screening at low potentials and the charge density of a single accelerated and converging species at high potentials. The quantity $C$, which is a function of the local potential and electric field, accounts for the increase in charge density as charged particles from a large area are attracted to a small region. The convergence formula was developed to fit the results of Langmuir and Blodgett [6] for current collection by a sphere. In a dense plasma, when the spacecraft velocity and Earth's magnetic field have minimal effect on the charge density within the sheath, this formula is appropriate.

Once Poisson's equation has been solved for space potentials, surface currents can be determined by tracking macroparticles either from a sheath edge or from the boundary of the computational space as appropriate.

When a sheath exists, it can be more convenient to track macroparticles from the sheath edge. Macroparticles can be created at the sheath edge and then tracked in the computed potentials and any specified magnetic fields to determine the current to each surface. In the computation, the sheath edge is a surface at a specified potential, for example, $\phi= \pm T \ln 2$. The justification for this choice is that because the attracted species is absorbed by the sheath, only the inward moving component is present, comprising half the ambient density. The repelled species, whose density satisfies $n(\phi)=n \exp (-|\phi / T|)$, must also be at half the ambient density, leading immediately to this sheath potential. The current for each macroparticle is the thermal current for the sheath area it represents. The simplest assumption for the initial velocity of the macroparticles is the average velocity of charged particles crossing the sheath edge, $\sqrt{\frac{2 e T}{\pi m}}$

The approximations made when tracking from a sheath are not valid when either the thermal distribution of particle 
velocities or the spacecraft velocity is important. In these situations macroparticles are tracked from the boundary of the computational grid. Enough macroparticles need to be tracked to capture the distribution function. The velocity and current carried by each macroparticle can be assigned stochastically or a specified portion of the distribution function can be explicitly specified. Macroparticles can be distributed so that they have approximately the same weight or macroparticles with less current can be used to represent the edges of the distribution function.

A geometric wake factor can be added to the charge density and sheath current formulas to account for spacecraft (and/or plasma) motion.

The stationary two-sphere example discussed above can be used to illustrate this type of model. The potentials shown in Fig. 2 were computed by Nascap-2k using the charge density formula of (5). The current to each sphere was computed both by tracking macroparticles from the sheath edge and by tracking particles from the boundary of the computational space and the sphere potentials were adjusted to find the potentials for which the net current is zero. The surface potentials of $+0.25 \mathrm{~V}$ and $-99.75 \mathrm{~V}$, shown in Fig. 2, are those that result when tracking from the sheath. This result is $20 \mathrm{~V}$ different than that obtained for the same sphere size and plasma properties using the analytic model of sheath current above.

When tracking from the sheath edge, the current to each sphere includes only the attracted species. When tracking macroparticles of both species from the boundary of the computational space, it is possible to find a floating potential with both spheres negative. For this geometry, the floating potentials of $-100.2 \mathrm{~V}$ and $-0.2 \mathrm{~V}$ were found. The $-0.2 \mathrm{~V}$ sphere collects both ions and the high energy tail of the electron phase space distribution function, which when combined with the ion collection of the $-100.2 \mathrm{~V}$ sphere gives zero net current.

As shown in Fig. 3, at lower density, the large (negative)

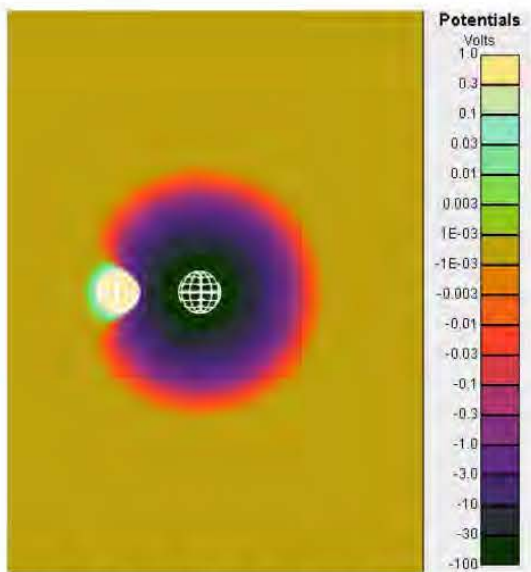

Fig. 2. Potentials on plane through center of stationary two-sphere object with spheres at their floating potentials, of $+0.25 \mathrm{~V}$ and $99.75 \mathrm{~V}$, computed by tracking macroparticles from the boundary of the computational space, in $10^{11} \mathrm{~m}^{-3}, 0.2 \mathrm{eV}$ plasma.
TABLE II. TWO-SPHERE FLOATING POTENTIALS (V) COMPUTED USING DIFFERENT TECHNIQUES

\begin{tabular}{|l|c|c|c|c|c|c|}
\hline \multicolumn{1}{|c|}{ Debye length (cm) } & \multicolumn{2}{c|}{1.05} & \multicolumn{2}{c|}{3.32} & \multicolumn{2}{c|}{10.5} \\
\hline Analytic currents, Sheath & -90.9 & +9.1 & -19.6 & +80.4 & & \\
\hline Analytic currents, Maxwellian & -99.6 & +0.4 & -99.6 & +0.4 & -99.6 & +0.4 \\
\hline Tracked currents from sheath & -99.75 & +0.25 & -98.1 & +1.9 & -86.1 & +13.9 \\
\hline Tracked currents from boundary & -100.2 & -0.2 & -98.7 & +1.3 & -88 & +12 \\
\hline
\end{tabular}

sheath gets larger, enveloping the positive sphere. In order to achieve current balance, the positive sphere is at a higher potential, here $13.9 \mathrm{~V}$, so that the positive potential peeks through and electrons can be collected. For this simple geometry, the ratio of the exposed sheath areas matches the ratio of plasma thermal currents.

The floating potentials for the two-sphere example for $10^{11} \mathrm{~m}^{-3}, 10^{10} \mathrm{~m}^{-3}$ and $10^{9} \mathrm{~m}^{-3}, 0.2 \mathrm{eV}$, oxygen plasmas are shown in Table II. The results using the Analytic Currents, Sheath model matches the other results best in the shortest Debye length case, for which it is expected to be most accurate. For this example, the results are not sensitive to whether the currents are tracked from the sheath edge or the boundary. When the spacecraft is moving and when the currents themselves are important (rather than just their relative values), it can be important to track the macroparticles from the boundary.

\section{Hybrid PIC}

When the spacecraft geometry is complex enough that analytic charge densities are inadequate, the charge density can be given by the sum of an ion density determined by tracking macroparticles and an electron density given by a Boltzmann function. The equilibrium electron density is Boltzmann for negative potentials in the absence of a potential barrier and nearly Boltzmann for small barriers.

As the ion macroparticles are used to give the ion charge density, they must be tracked from the computational boundary.
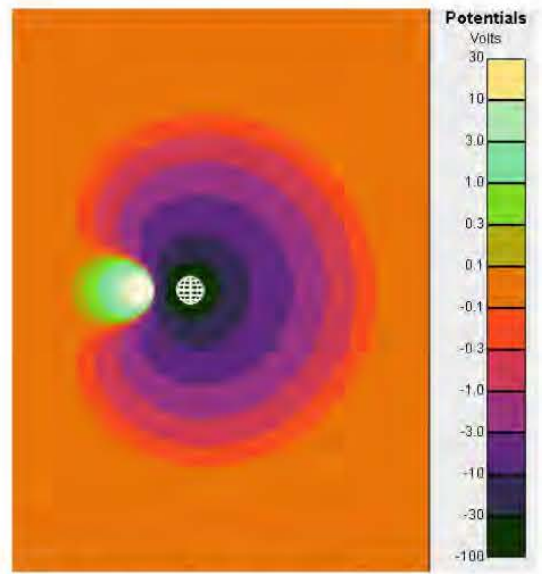

Fig. 3. Potentials on plane through center of two-sphere object with spheres at their floating potentials of $+13.9 \mathrm{~V}$ and $-86.1 \mathrm{~V}$, in $10^{9} \mathrm{~m}^{-3}$, $0.2 \mathrm{eV}$ plasma $\left(\lambda_{D}=10.5 \mathrm{~cm}\right)$ 
To get self-consistent potentials and ion densities, there are two approaches. The ions can be tracked throughout the grid and then the resultant potentials computed and the process iterated, possibly including sharing of ion densities between iterations. Alternatively, a more traditional PIC approach can be used in which the computational space is filled with ion macroparticles. The macroparticles are tracked for a short time, the potentials are computed, additional ions are injected from the computational boundary, and the process repeated until all transients disappear. In both cases, the time required to reach equilibrium depends on how close the initial potentials are to the potentials for zero current. As mentioned above for electron tracking to obtain surface currents, the initial velocities of the macroparticles used to represent the velocity distribution of phase space can be assigned stochastically or a specified portion of the distribution function can be explicitly specified. The accuracy of the potential solution (and therefore the resulting currents and zero current surface potentials) is limited by the adequacy of sampling of the ion phase space distribution function.

The tracked ion macroparticles can be used to compute the surface currents as well as the spatial charge density. The electron surface current can be computed using any of the analytic or tracked approaches described above.

The importance of velocity resolution can be illustrated by considering the floating potential of an isolated, conducting sphere. While an isolated sphere or cube is a favorite for testing charging codes, its symmetry and low potential make it numerically challenging. We use a stationary, isolated $1 \mathrm{~cm}$ radius conductive sphere. In a $10^{10} \mathrm{~m}^{-3}$ to $10^{12} \mathrm{~m}^{-3}, 0.1 \mathrm{eV}$. oxygen plasma, the Debye length of $0.235 \mathrm{~cm}$ to $2.35 \mathrm{~cm}$ is comparable to the sphere size. Above we obtained a floating potential of $-0.5 \mathrm{I} \mathrm{V}$ in the planar limit and $-0.36 \mathrm{~V}$ in the orbit-limited current collection limit. Table III gives the floating potential of this sphere computed using an analytic charge density and Hybrid PIC techniques with various representations of the velocity distribution.

First, we use the analytic charge density function when computing the space potentials, track a distribution of ion macroparticles in those potentials from the boundary of the computational space, and use the Boltzmann relation to specify the electron current. When the Debye length is larger than the sphere, as expected, the orbit limited result is obtained. In a shorter Debye length plasma, the surface potential is shielded by the attracted ions, so that ion collection is less than orbitlimited, and the sphere floats more negative. Fig. 4 shows the potential in a plane through the center of the sphere.

If the same calculation is performed using the Hybrid PIC approach with a minimal distribution of initial velocity values of the ion macroparticles, the resulting sphere potential is slightly more negative and the space potentials seen in Fig. 5 result. In this calculation, the thermal velocity distribution is represented by eight evenly weighted macroparticles with the average thermal velocity plus a component to represent the thermal distribution about a randomly generated coordinate axis. That the lowest potential contour is somewhat square, like
TABLE III. FLOATING POTENTIAL (V) OF SPHERE COMPUTED USING DIFFERENT TECHNIQUES

\begin{tabular}{|l|c|c|c|}
\hline Debye length $(\mathrm{cm})$ & 0.24 & 0.74 & 2.35 \\
\hline $\begin{array}{l}\text { Analytic charge density, tracked ion current and } \\
\text { analytic electron current }\end{array}$ & -0.40 & -0.37 & -0.36 \\
\hline Hybrid PIC with poor ion velocity resolution & & -0.40 & \\
\hline Hybrid PIC with better ion velocity resolution & -0.38 & -0.37 & -0.36 \\
\hline $\begin{array}{l}\text { Hybrid PIC and track electrons with poor velocity } \\
\text { resolution for currents }\end{array}$ & -0.33 & -0.30 & -0.28 \\
\hline $\begin{array}{l}\text { Hybrid PIC and track electrons with better } \\
\text { velocity resolution }\end{array}$ & -0.37 & -0.34 & -0.33 \\
\hline
\end{tabular}

the computation grid, rather than circular, like the sphere, suggests that the results may not be correct. Adding additional ion macroparticles with a wider range of initial velocities. shown in Fig 6 gives a result similar to the analytic charge density calculation (which for this calculation is expected to give a highly reliable result). A total of 64 macroparticles were created at each initial location. In each of the three spatial directions, these macroparticles represent the $10 \%$ of ions moving fastest in the negative direction, the other $40 \%$ moving in the negative direction, $10 \%$ of the ions moving fastest in the positive direction. and the other $40 \%$ moving in the positive direction.

When the electron current is computed using macroparticles, the adequacy of the representation of the high energy portion of phase space becomes very important, as can be seen by comparing the floating potentials computed with poor and better resolution. In the first of the calculations in which the electron current is determined by tracking electron macroparticles, the electron thermal distribution is represented in the same manner as the ion distribution. This is inadequate as only a small fraction of the electrons have enough energy to reach the sphere. In the better resolution calculation, a similar scheme for representing the distribution is used. At each emitting point on the boundary, 1,000 macroparticles are created. The electrons distribution is divided into those with the $2.5 \%$ most negative velocities, those with the next $2.5 \%$ most negative, those with the next $5 \%$ most negative, those with the next $5 \%$ most negative, the remaining $35 \%$ with negative velocities, and the same for positive velocities.

\section{PIC}

The first principles approach is to use macroparticle tracking of all species to determine the charge density for Poisson's equation as well as to determine the surface currents. This requires even more computer time and a great deal of skill to avoid numeric pathologies. There is an extensive literature of techniques to help speed solutions and improve stability developed by researchers modelling dynamic plasma conditions. The calculation is inherently dynamic, so averaging and smoothing are generally needed to determine the steadystate solution.

This approach becomes necessary when investigating novel effects and exploring dynamic conditions. 


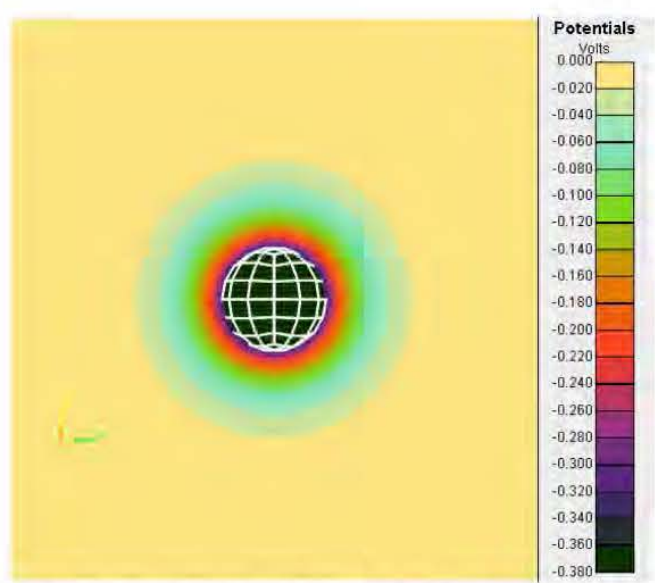

Fig. 4. Potentials in a plane through the center of a sphere computed using the analytic space charge density of (5) at the floating potential of $-0.37 \mathrm{~V}$ in a $10^{11} \mathrm{~m}^{-3}, 0.1 \mathrm{eV}$ plasma.

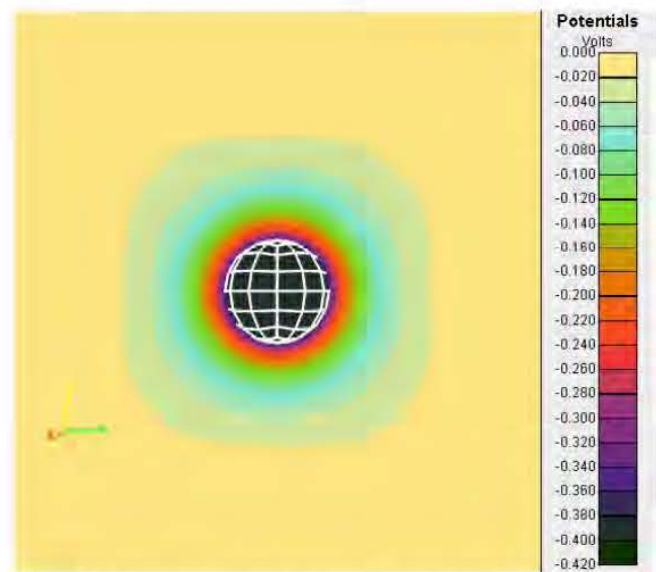

Fig. 5. Potentials in a plane through the center of a sphere computed using the Hybrid PIC method with minimal velocity resolution at the floating potential of $-0.40 \mathrm{~V}$ in a $10^{11} \mathrm{~m}^{-3}, 0.1 \mathrm{eV}$ plasma.

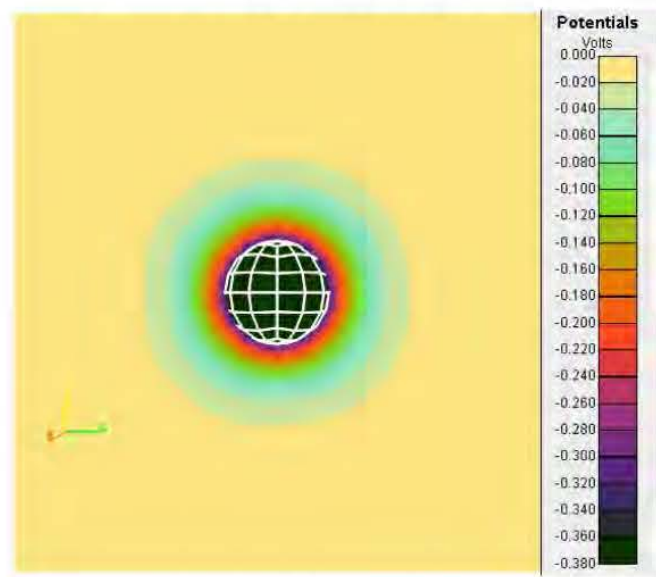

Fig. 6. Potentials in a plane through the center of a sphere computed using the Hybrid PIC method with better velocity resolution at the floating potential of $-0.40 \mathrm{~V}$ in a $10^{11} \mathrm{~m}^{-3}, 0.1 \mathrm{eV}$ plasma.

\section{CONCLUSIONS AND IMPLICATIONS}

We have surveyed a selection of methods to compute currents when solving for floating potentials of surfaces in dense, cold plasma such as observed in low Earth orbit. The methods range from analytic to full PIC. Analytical methods are easy to apply and give results quickly. However, they are only applicable when the underlying approximations are valid. At the other extreme, full PIC calculations are applicable to complex geometry and dynamic conditions. However, they require large investments of computational resources and a great deal of skill to obtain reliable results.

In practice, computations generally combine analytic methods with PIC techniques. With an understanding of the underlying approximations, reliable results can be obtained with the minimum necessary resources.

The extent to which the approximations are valid is highly dependent on the system and the question at hand. To gain confidence in the results, it is best to use the simplest applicable models to gain insight, and then use more complex approaches to verify the applicability of the approximation. When calculations are dependent on adequate resolution, they can be checked by verifying that the results are the same with improved resolution.

\section{REFERENCES}

[1] H. Goldstein, Classical Mechanics, Addison-Wesley Publishing Company, p. 68, 1950

[2] I. Katz, V.A. Davis, M.J. Mandell, D.E. Parks, B.M. Gardner, J.M. Hilton, J. Minor, D.L. Cooke, An Auroral Charging Model in the Spacecraft Charging Interactive Handbook, Aerospace Sciences Meeting, AIAA 2001-0956, 2001.

[3] B.M. Gardner, G. Jongeward, B. Kuharski, K. Wilcox, T. Rankin, J Roche, The Environment Workbench: A Design Tool for the International Space Station, Aerospace Sciences Meeting, Reno, NV, AIAA 95-0599, 1995.

[4] L.W. Parker, Plasmasheath-Photosheath Theory for Large High-Voltage Space Structures, Space Systems and Their Interactions with Earth's Space Environment, Eds. H.B. Garrett and C.P. Pike, in Progress in Astronautics and Aeronautics, Vol. 71, M. Summerfield Series Editor, p. $477,1980$.

[5] V.A. Davis and M.J. Mandell, Plasma Interactions with Spacecraft. Volume 2, Nascap-2k Scientific Documentation for Version 4.1, AFRLRV-PS-TR-2011-0089, Vol II, 2011.

[6] I. Langmuir, K.B. Blodgett, Currents limited by space charge between concentric spheres, Phys. Rev., 24, p. 49, 1924. 


\section{DISTRIBUTION LIST}

\section{DTIC/OCP}

8725 John J. Kingman Rd, Suite 0944

Ft Belvoir, VA 22060-6218

$1 \mathrm{cy}$

AFRL/RVIL

Kirtland AFB, NM 87117-5776

2 cys

Official Record Copy

AFRL/RVBXR/Adrian Wheelock 1 cy 
This page is intentionally left blank. 